\title{
Trivializations of differential cocycles
}

\author{
Corbett Redden
}

Received: 3 December 2012 / Accepted: 15 October 2013 / Published online: 7 November 2013

(C) Tbilisi Centre for Mathematical Sciences 2013

\begin{abstract}
Associated to a differential character is an integral cohomology class, referred to as the characteristic class, and a closed differential form, referred to as the curvature. The characteristic class and curvature are equal in de Rham cohomology, and this is encoded in a commutative square. In the Hopkins-Singer model, where differential characters are equivalence classes of differential cocycles, there is a natural notion of trivializing a differential cocycle. In this paper, we extend the notion of characteristic class, curvature, and de Rham class to trivializations of differential cocycles. These structures fit into a commutative square, and this square is a torsor for the commutative square associated to characters with degree one less. Under the correspondence between degree two differential cocycles and principal circle bundles with connection, we recover familiar structures associated to global sections.
\end{abstract}

Keywords Differential cohomology $\cdot$ Chern-Simons invariants

Mathematics Subject Classification (2000) Primary 53C08; Secondary 58J28

\section{Introduction}

The differential cohomology groups $\check{H}^{k}(M ; \mathbb{Z})$, defined for a smooth manifold $M$, are a hybrid of ordinary integral cohomology and differential forms. More precisely, there is a functorially defined commutative square

Communicated by Thomas Schick.

C. Redden $(\varangle)$

Department of Mathematics, C. W. Post Campus of Long Island University,

720 Northern Blvd, Brookville, NY 11548, USA

e-mail: corbett.redden@liu.edu 


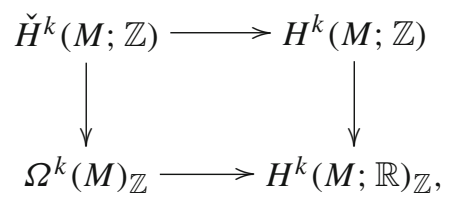

where $\Omega^{k}(M)_{\mathbb{Z}} \subset \Omega^{k}(M)$ are closed smooth $k$-forms with $\mathbb{Z}$-periods, and $H^{k}(M ; \mathbb{R})_{\mathbb{Z}} \subset H^{k}(M ; \mathbb{R})$ is the image of $H^{k}(M ; \mathbb{Z}) \rightarrow H^{k}(M ; \mathbb{R})$. All four homomorphisms are surjective. To an element in $\check{H}^{k}(M ; \mathbb{Z})$, the induced elements in $H^{k}(M ; \mathbb{Z}), \Omega^{k}(M)$, and $H^{k}(M ; \mathbb{R})$ are referred to as the characteristic class, curvature, and de Rham class, respectively. While the characteristic class and curvature do not completely determine an element in $\check{H}^{k}(M ; \mathbb{Z})$, they provide a useful way of compartmentalizing the differential cohomology groups.

Cocycle models for differential cohomology allow one to define trivializations. This idea is not new; for example, Hopkins-Singer emphasize this point in [8], and trivializations play a fundamental role in Freed's paper [4]. We use the definition that generalizes the notion of trivializing an $\mathbb{R} / \mathbb{Z}$-bundle with connection by an arbitrary global section, as opposed to a flat global section. In general, a differential cocycle $\check{x} \in \check{\mathcal{H}}^{k}(M ; \mathbb{Z})$ admits a trivialization if and only if its characteristic class vanishes. For $\breve{x}$ trivializable, we let $\mathcal{T}_{\text {geo }}(x)$ denote the category of trivializations. The set of isomorphism classes, denoted $T_{\mathrm{geo}}(x)$, is a torsor (principal homogeneous space) for $\breve{H}^{k-1}(M ; \mathbb{Z})$. Our subscript "geo" is due to the fact that the classes contain local geometric information. Because the sets $T_{\text {geo }}(\check{x})$ are torsors and not groups, they are intrinsically subtle. Furthermore, their definition involves the actual cocycle $\check{x}$ as opposed to the differential character in $\check{H}^{k}(M ; \mathbb{Z})$.

The motivation for this paper is to understand classes in $T_{\text {geo }}(\check{x})$ more explicitly. We accomplish this by extending the notion of characteristic class, curvature, and de Rham class to trivializations of differential cocycles. We define a category $\mathcal{T}_{\text {top }}(\check{x})$, whose isomorphism classes are denoted $T_{\text {top }}(\check{x})$, along with sets $T_{\text {curv }}(\check{x})$ and $T_{\mathrm{dR}}(\check{x})$. These sets are where the characteristic class, curvature, and de Rham class of a trivialization live. While the sets $T_{\text {geo }}(\check{x})$ and $T_{\text {top }}(\check{x})$ depend on the specific cocycle $\check{x}$, the sets $T_{\text {curv }}(\check{x})$ and $T_{\mathrm{dR}}(\check{x})$ are defined using only the equivalence class $[\check{x}] \in \check{H}^{k}(M ; \mathbb{Z})$ and are subsets of more familiar groups. The set $T_{\text {curv }}(\check{x}) \subset \Omega^{k-1}(M)$ is the inverse image of $[\check{x}]$ in the homomorphism $\Omega^{k-1}(M) \rightarrow \check{H}^{k}(M ; \mathbb{Z})$ given by integrating and quotienting $\bmod \mathbb{Z}$. Elements of $T_{\mathrm{dR}}(\check{x})$ are lifts of the differential character from $\operatorname{Hom}\left(Z_{k-1}(M), \mathbb{R} / \mathbb{Z}\right)$ to $\operatorname{Hom}\left(Z_{k-1}(M), \mathbb{R}\right)$.

Our notation is justified by Theorem 3.11 , which states that the sets $T_{\bullet}(\check{x})$ naturally fit into a commutative square that is a torsor for the fundamental square (1.1) associated to $\check{H}^{k-1}(M ; \mathbb{Z})$.
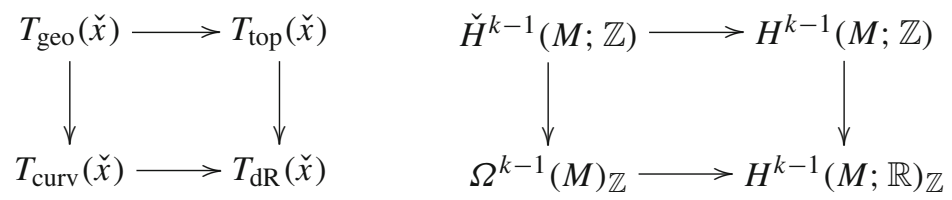
In other words, the sets $T_{\text {geo }}(\check{x}), T_{\text {top }}(\check{x}), T_{\text {curv }}(\check{x}), T_{\mathrm{dR}}(\check{x})$ are torsors over $\check{H}^{k-1}(M ; \mathbb{Z}), H^{k-1}(M ; \mathbb{Z}), \Omega^{k-1}(M)_{\mathbb{Z}}, H^{k-1}(M ; \mathbb{R})_{\mathbb{Z}}$, respectively, and there are natural surjective equivariant maps making the square on the left into a commutative square.

The paper is organized as follows. Section 2 contains standard background information on Cheeger-Simons differential characters [3] and the Hopkins-Singer model for differential ordinary cohomology [8]. The one part not already in the literature is the exact sequence for the change of coefficients in differential cohomology; it appears in Proposition 2.11 and Eq. 2.7. Section 3 is the heart of the paper. We define the the sets $T_{\bullet}(\check{x})$ and show they fit into a commutative square of torsors. Section 3.2 shows how the Hodge decomposition of forms fits into differential cohomology and trivializations. On a closed manifold, a Riemannian metric induces a right inverse $T_{\text {curv }}(\check{x}) \leftarrow T_{\mathrm{dR}}(\check{x})$ that is equivariant with respect to the usual right inverse $\Omega^{k-1}(M)_{\mathbb{Z}} \leftarrow H^{k-1}(M ; \mathbb{R})_{\mathbb{Z}}$ given by projecting onto harmonic forms. However, we prove this cannot be refined to give a natural right inverse for the characteristic class or curvature map. Sections 3.3 and 3.4 show that the sets $T_{\bullet}(\check{x})$ behave as expected with respect to products and integration. In Sect. 4, we consider the degree two case. When a cocycle $\check{x} \in \check{\mathcal{H}}^{2}(M ; \mathbb{Z})$ represents a principal $\mathbb{R} / \mathbb{Z}$-bundle with connection $(P, \Theta) \stackrel{\pi}{\rightarrow} M$, the sets $T_{\text {geo }}(\check{x}), T_{\text {top }}(\check{x}), T_{\text {curv }}(\check{x})$, and $T_{\mathrm{dR}}(\check{x})$ are naturally isomorphic to the following sets, respectively: global sections of $P$, homotopy classes of global sections of $P$, connection forms for $\Theta$ globally defined on $M$, and lifts of values of the holonomy to $\mathbb{R}$.

There are various reasons to consider trivializations of differential cocycles. Many geometric structures arising in theoretical physics can be described in terms of differential (generalized) cohomology or trivializations of differential cocycles. As explained in [4], trivializations in differential ordinary cohomology are a natural generalization of abelian gauge fields in the presence of a magnetic current, and they often give a mathematical description of anomaly cancellation mechanisms. In another example, trivializations play an important role in formulating $T$-duality within the context of twisted differential $K$-theory [9]. Also, trivializations are useful when considering lifts of structure groups. Given a principal $S O(n)$-bundle with connection, lifts to a $\operatorname{Spin}^{c}(n)$-bundle with connection are equivalent to trivializations of a differential refinement of the $W_{3}$ characteristic class [11]. Similarly, given a principal $\operatorname{Spin}(n)$ bundle with connection, one can formulate geometric string structures as trivializations of a cocycle for the character $\frac{\check{p}_{1}}{2}$, as done in [12] using the language of bundle 2-gerbes.

We should note that there exist different cocycle models for various differential cohomology theories. For example, Deligne-Beilinson cohomology [1] and HarveyLawson spark complexes [7] give alternate constructions of the groups $\check{H}^{*}(M ; \mathbb{Z})$, and Hopkins-Singer define a differential refinement for any generalized cohomology theory [8]. While our commutative square of torsors can be defined in other cocycle models, we refrain from doing so in this paper so that the discussion remains explicit and simple. 


\section{Review of differential cohomology}

We always work in the category of smooth manifolds; objects are smooth manifolds and morphisms are smooth maps. We also work with the smooth singular chain and cochain complexes, $C_{*}(M)$ and $C^{*}(M ;-)$, and we denote cycles and cocycles by $Z_{*}(M)$ and $Z^{*}(M ;-)$. The bracket $\langle\cdot, \cdot\rangle$ denotes the pairing between cochains and chains.

Let $V$ be a finite-dimensional vector space, and $\Lambda \subset V$ a completely disconnected subgroup; i.e. the only connected components of $\Lambda$ are points. Define $H^{k}(M ; V)_{\Lambda} \subset$ $H^{k}(M ; V)$ to be the image of $H^{k}(M ; \Lambda) \rightarrow H^{k}(M ; V)$, and let $\Omega^{k}(M ; V)_{\Lambda} \subset$ $\Omega^{k}(M ; V)=\Omega^{k}(M) \otimes V$ denote the closed smooth $V$-valued $k$-forms on $M$ with periods in $\Lambda$. In other words, $\omega \in \Omega^{k}(M ; V)_{\Lambda}$ if and only if $\int_{z} \omega \in \Lambda$ for all smooth cycles $z \in Z_{k}(M)$. Equivalently, $\Omega^{k}(M ; V)_{\Lambda}$ is defined so that $\frac{\Omega^{k}(M ; V)_{\Lambda}}{d \Omega^{k-1}(M ; V)} \cong$ $H^{k}(M ; V)_{\Lambda}$.

We now introduce the differential (ordinary) cohomology groups $\check{H}^{*}(M ; \Lambda)$ using the models ${ }^{1}$ from Cheeger-Simons [3] and Hopkins-Singer [8]. The first model uses differential characters; it is the most geometric and emphasizes the idea of holonomy. The second model is given as the cohomology of a cochain complex and allows us to define trivializations.

Definition 2.1 ([3]) The group of differential characters of degree $k,{ }^{2}$ with values in $V / \Lambda$, is

$\check{H}^{k}(M ; \Lambda):=\left\{\begin{array}{l|l}\chi \in \operatorname{Hom}\left(Z_{k-1}(M), V / \Lambda\right) & \begin{array}{l}\exists \omega \in \Omega^{k}(M ; V) \text { satisfying } \\ \langle\chi, \partial z\rangle=\int_{z} \omega \bmod \Lambda \forall z \in C_{k}(M)\end{array}\end{array}\right.$.

Proposition 2.2 ([3]) The groups $\check{H}^{k}(M ; \Lambda)$ fit into the commutative square

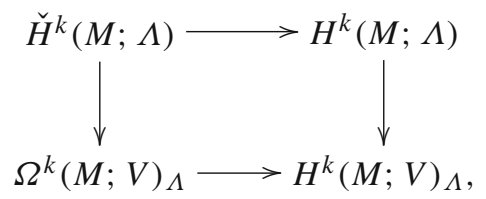

and these homomorphisms determine the short exact sequences

$$
\begin{aligned}
0 & \rightarrow H^{k-1}(M ; V / \Lambda) \rightarrow \check{H}^{k}(M ; \Lambda) \rightarrow \Omega^{k}(M ; V)_{\Lambda} \rightarrow 0 \\
0 & \rightarrow \frac{\Omega^{k-1}(M ; V)}{\Omega^{k-1}(M ; V)_{\Lambda}} \rightarrow \check{H}^{k}(M ; \Lambda) \rightarrow H^{k}(M ; \Lambda) \rightarrow 0 \\
0 & \rightarrow \frac{H^{k-1}(M ; V)}{H^{k-1}(M ; V)_{\Lambda}} \rightarrow \check{H}^{k}(M ; \Lambda) \rightarrow A^{k}(M ; \Lambda) \rightarrow 0
\end{aligned}
$$

\footnotetext{
${ }^{1}$ While there exist many different explicit models for $\check{H}^{*}(M ; \Lambda)$, they are all isomorphic, a fact proven elegantly in [10].

2 This grading is different than the one originally used by Cheeger-Simons.
} 
Here, $A^{k}(M ; \Lambda)$ is defined to be the the pullback in diagram (2.1). The induced homomorphism $H^{k-1}(M ; V / \Lambda) \rightarrow H^{k}(M ; \Lambda)$, given by (2.2) and (2.3), equals minus the Bockstein homomorphism. The homomorphism $\Omega^{k-1}(M ; V) / \Omega^{k-1}(M ; V)_{\Lambda} \rightarrow$ $\Omega^{k}(M ; V)_{\Lambda}$, induced from (2.3) and (2.2), equals the de Rham differential $d$.

Remark 2.3 The notation $\check{H}^{k}(M ; \Lambda)$ may seem a bit confusing, since the definition involves both $V$ and $\Lambda$. However, we view $\Lambda$ not as an abstract group, but as a subgroup of the vector space $V$.

Remark 2.4 While $V=\mathbb{R}$ and $\Lambda=\mathbb{Z}$ in most examples and in much of the cited literature, the definitions and properties easily generalize. The key point is that when $\Lambda$ is totally disconnected, the natural homomorphism

$$
\Omega^{k}(M ; V) \rightarrow C^{k}(M ; V / \Lambda),
$$

given by integrating and quotienting modulo $\Lambda$, is injective. This fact can be easily proven pointwise by integrating a $k$-form over an arbitrarily small disk.

Remark 2.5 One can also replace $\Lambda \subset V$ by a totally disconnected subgroup of a $\mathbb{Z}$-graded vector space $\Lambda \subset \mathcal{V}$. This is useful when considering "Chern characters" in differential generalized cohomology. For example, the ordinary Chern character in $K$-theory can be refined to a natural transformation

$$
\check{K}^{*}(M) \stackrel{\check{\mathrm{ch}}}{\longrightarrow} \check{H}^{*}\left(M ; \mathbb{Q}\left[\beta^{ \pm}\right]\right)
$$

where $\mathbb{Q}\left[\beta^{ \pm}\right] \subset \mathbb{R}\left[\beta^{ \pm}\right]$and $|\beta|=2([2])$.

The homomorphism $\check{H}^{k}(M ; \Lambda) \rightarrow \Omega^{k}(M ; V)$ is given by the form $\omega$ in Definition 2.1. We call this form the curvature of $\chi$, and we say that $\chi$ is flat if $\omega=0$. The induced class in $H^{k}(M ; \Lambda)$ is referred to as the characteristic class of $\chi$. Diagram (2.1) implies that the curvature equals the characteristic class in de Rham cohomology. We refer to the value $\langle\chi, z\rangle$ as the holonomy of $\chi$ along $z \in Z_{k-1}(M)$. This terminology is made particularly clear in the second example below.

Example 2.6 There is a canonical isomorphism

$$
C^{\infty}(M, \mathbb{R} / \mathbb{Z}) \cong \check{H}^{1}(M ; \mathbb{Z}) \subset \operatorname{Hom}\left(Z_{0}(M), \mathbb{R} / \mathbb{Z}\right)
$$

To a differential character given by a function $f \in C^{\infty}(M, \mathbb{R} / \mathbb{Z})$, the characteristic class is the homotopy class

$$
[f] \in[M, \mathbb{R} / \mathbb{Z}] \cong[M, K(\mathbb{Z}, 1)] \cong H^{1}(M ; \mathbb{Z}),
$$

and the curvature is the derivative $d f \in \Omega^{1}(M)$.

Example 2.7 Let $(P \stackrel{\pi}{\rightarrow} M, \Theta)$ be a principal $\mathbb{R} / \mathbb{Z}$-bundle with connection $\Theta$. The holonomy of $\Theta$ is invariant under gauge transformations, and it associates an element 
in $\mathbb{R} / \mathbb{Z}$ to any loop in $M$. This defines an isomorphism between gauge equivalence classes of $\mathbb{R} / \mathbb{Z}$-bundles with connection and differential characters of degree two

$$
\{\mathbb{R} / \mathbb{Z} \hookrightarrow(P, \Theta) \rightarrow M\}_{/ \sim} \stackrel{\cong}{\longrightarrow} \check{H}^{2}(M ; \mathbb{Z})
$$

The characteristic class of $[(P, \Theta)] \in \check{H}^{2}(M ; \mathbb{Z})$ is the cohomology class that classifies $P$, and the character's curvature equals the connection's curvature $d \Theta$. More generally, $\breve{H}^{2}(M ; \Lambda)$ classifies principal $V / \Lambda$-bundles with connection when $V / \Lambda$ is a Lie group. This fact is used in the discussion of $T$-duality in [9].

While equating two bundles that are gauge equivalent makes many things simpler, one loses important information. For example, local cutting and gluing constructions can not be performed on isomorphism classes. This is due to the existence of non-trivial automorphisms. In general, one should consider the category of principal $\mathbb{R} / \mathbb{Z}$-bundles with connection on $M$, where the morphisms are connection preserving bundle maps.

For the same reasons, it is useful to consider a category of differential cocycles $\check{\mathcal{H}}^{k}(M ; \Lambda)$ whose set of equivalence classes $\pi_{0}\left(\check{\mathcal{H}}^{k}(M ; \Lambda)\right)$ is canonically isomorphic to the groups $\check{H}^{k}(M ; \Lambda)$. We choose to use the following cochain model, constructed by Hopkins-Singer. The category $\check{\mathcal{H}}^{2}(M ; \mathbb{Z})$ is equivalent to the category of $\mathbb{R} / \mathbb{Z}$ bundles with connection on $M$, as explained in Sect. 4.

We will frequently use the following general method of associating a category to a cochain complex. The terminology arises from simplicial methods and the DoldKan correspondence, but we only need basic homological algebra. Given a cochain complex of abelian groups $\left(C^{*}, d\right)$, the fundamental groupoid

$$
\pi_{\leq 1}\left(C^{k-2} \stackrel{d}{\rightarrow} C^{k-1} \stackrel{d}{\rightarrow} C^{k}\right)
$$

is the category whose objects are elements of $C^{k}$, and whose morphisms between $x_{1}, x_{2} \in C^{k}$ are

$$
\operatorname{Hom}\left(x_{1}, x_{2}\right)=\left\{y \in C^{k-1} \mid d y=x_{2}-x_{1}\right\} / d C^{k-2} \subset C^{k-1} / d C^{k-2} .
$$

The abelian group structure on $C^{*}$ induces an abelian group structure on the set of isomorphism classes $C^{k} / d C^{k-1}$, and the automorphisms of any object $x$ are naturally identified with $H^{k-1}\left(C^{*}\right)$. One can replace $C^{k}$ by a subset $A^{k} \subset C^{k}$ that is closed under the addition of $d C^{k-1}$, but the set of isomorphism classes will not necessarily be a group.

Definition 2.8 ([8]) The differential cochain complex $\left(\check{C}(n)^{*}(M ; \Lambda), d\right)$ is defined as

$$
\check{C}(n)^{k}(M ; \Lambda):= \begin{cases}C^{k}(M ; \Lambda) \times C^{k-1}(M ; V) \times \Omega^{k}(M ; V) & k \geq n \\ C^{k}(M ; \Lambda) \times C^{k-1}(M ; V) & k<n\end{cases}
$$

with differential 


$$
\begin{aligned}
d(c, h, \omega) & =(\delta c, \omega-c-\delta h, d \omega) \\
d(c, h) & = \begin{cases}(\delta c,-c-\delta h, 0) & (c, h) \in \check{C}(k)^{k-1}(M ; \Lambda) \\
(\delta c,-c-\delta h) & \text { otherwise. }\end{cases}
\end{aligned}
$$

Differential cocycles are cocycles in this complex, and $\breve{H}(n)^{k}(M ; \Lambda)$ is the $k$ th cohomology; i.e.

$$
\begin{aligned}
& \check{Z}(n)^{k}(M ; \Lambda)=\left\{\check{x} \in \check{C}(n)^{k}(M ; \Lambda) \mid d \check{x}=0\right\}, \\
& \check{H}(n)^{k}(M ; \Lambda)=\check{Z}(n)^{k}(M ; \Lambda) / d \check{C}(n)^{k-1}(M ; \Lambda) .
\end{aligned}
$$

For $n \neq k$, the cohomology groups of the differential cochain complex are naturally isomorphic to ordinary cohomology groups. When $n=k$, we obtain the differential cohomology groups:

$$
\check{H}(n)^{k}(M ; \Lambda) \cong \begin{cases}H^{k}(M ; \Lambda) & k>n \\ \check{H}^{k}(M ; \Lambda) & k=n \\ H^{k-1}(M ; V / \Lambda) & k<n .\end{cases}
$$

Definition 2.9 ([8]) The category of differential $k$-cocycles $\check{\mathcal{H}}^{k}(M ; \Lambda)$ is the fundamental groupoid

$$
\check{\mathcal{H}}^{k}(M ; \Lambda):=\pi_{\leq 1}\left(\check{C}(k)^{k-2}(M ; \Lambda) \stackrel{d}{\rightarrow} \check{C}(k)^{k-1}(M ; \Lambda) \stackrel{d}{\rightarrow} \check{Z}(k)^{k}(M ; \Lambda)\right) .
$$

In terms of objects and morphisms, this means

$$
\begin{aligned}
\operatorname{Ob~} \check{\mathcal{H}}^{k}(M ; \Lambda)= & \check{Z}(k)^{k}(M ; \Lambda), \\
\operatorname{Hom}\left(\check{x}_{1}, \check{x}_{2}\right)= & \left\{\check{y} \in \check{C}(k)^{k-1}(M ; \Lambda) \mid d \check{y}=\check{x}_{2}-\check{x}_{1}\right\} / d \check{C}(k)^{k-2}(M ; \Lambda) \\
& \subset \check{C}(k)^{k-1}(M ; \Lambda) / d \check{C}(k)^{k-2}(M ; \Lambda) .
\end{aligned}
$$

The set of isomorphism classes is an abelian group

$$
\pi_{0}\left(\check{\mathcal{H}}^{k}(M ; \Lambda)\right) \cong \check{H}^{k}(M ; \Lambda)
$$

and we denote an object $\check{x}=(c, h, \omega) \in \check{Z}(k)^{k}(M ; \Lambda)$ by

$$
\check{x} \in \check{\mathcal{H}}^{k}(M ; \Lambda) \text {. }
$$

From the triple $\check{x}=(c, h, \omega), c$ determines the characteristic class, $h$ determines the holonomy, and $\omega$ is the curvature. To see this explicitly, a differential cocycle $\check{x}$ is a triple 


$$
\check{x}=(c, h, \omega) \in C^{k}(M ; \Lambda) \times C^{k-1}(M ; V) \times \Omega^{k}(M ; V)
$$

satisfying

$$
\delta c=0, \quad \delta h=\omega-c, \quad d \omega=0 .
$$

In other words, $c$ and $\omega$ are closed, and the choice of $h$ specifies the equivalence $[c]=[\omega] \in H^{k}(M ; V)$ at the cochain level. One easily checks that the standard commutative square (2.1) is induced by the obvious maps below.
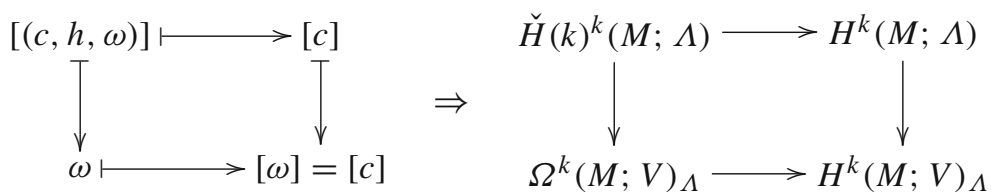

Furthermore, the canonical isomorphism from $\check{H}(k)^{k}(M ; \Lambda)$ to the differential characters $\check{H}^{k}(M ; \Lambda)$ is given by

$$
\begin{aligned}
\check{H}(k)^{k}(M ; \Lambda) & \stackrel{\cong}{\longrightarrow} \check{H}^{k}(M ; \Lambda) \\
{[(c, h, \omega)] } & \longmapsto\langle h, \bullet\rangle \bmod \Lambda .
\end{aligned}
$$

In the future, we will not distinguish between $\check{H}(k)^{k}(M ; \Lambda)$ and $\check{H}^{k}(M ; \Lambda)$.

Because the group $H^{k}(M ; V)_{\Lambda}$ appears frequently, it is worthwhile noting the following.

Lemma 2.10 There is a natural isomorphism

$$
H^{k}(M ; V)_{\Lambda} \cong \operatorname{Hom}\left(H_{k}(M), \Lambda\right)
$$

Proof The functor $\operatorname{Hom}\left(H_{k}(M),-\right)$ is left-exact, so the inclusion $\Lambda \hookrightarrow V$ naturally induces an injective homomorphism

$$
\operatorname{Hom}\left(H_{k}(M), \Lambda\right) \hookrightarrow \operatorname{Hom}\left(H_{k}(M), V\right)
$$

That $V$ is torsion-free implies $\operatorname{Ext}(-, V)=0$. Combining these facts with with the universal coefficient theorem gives the following sequences.

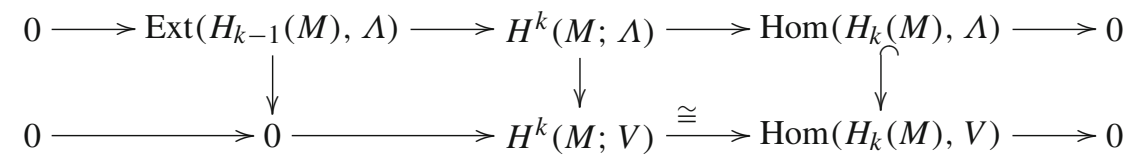

Therefore, the image of $H^{k}(M ; \Lambda) \rightarrow H^{k}(M ; V) \cong \operatorname{Hom}\left(H_{k}(M), V\right)$ is equal to the image of $\operatorname{Hom}\left(H_{k}(M), \Lambda\right)$. 


\subsection{Change of coefficients}

For spacing purposes, we use the notation $\check{H}^{k}(\Lambda)$ in this subsection to refer to the differential cohomology functor. In other words, $\check{H}^{k}(\Lambda)$ denotes the groups $\check{H}^{k}(M ; \Lambda)$ that are functorially associated to $M$.

Let $\Lambda_{1} \stackrel{i}{\hookrightarrow} \Lambda_{2} \rightarrow V$ be an inclusion of totally disconnected subgroups of $V$. This induces homomorphisms

$$
\check{H}^{k}\left(\Lambda_{1}\right) \stackrel{\check{i}}{\longrightarrow} \check{H}^{k}\left(\Lambda_{2}\right)
$$

The short exact sequences

$$
0 \rightarrow \Lambda_{1} \stackrel{i}{\rightarrow} \Lambda_{2} \rightarrow \Lambda_{2} / \Lambda_{1} \rightarrow 0 \quad \text { and } \quad 0 \rightarrow \Lambda_{2} / \Lambda_{1} \rightarrow V / \Lambda_{1} \rightarrow V / \Lambda_{2} \rightarrow 0
$$

also give rise to the long exact sequences

$$
\begin{aligned}
& \cdots \rightarrow H^{k}\left(\Lambda_{1}\right) \rightarrow H^{k}\left(\Lambda_{2}\right) \rightarrow H^{k}\left(\Lambda_{2} / \Lambda_{1}\right) \stackrel{\beta}{\rightarrow} H^{k+1}\left(\Lambda_{1}\right) \rightarrow \cdots, \\
& \cdots \rightarrow H^{k}\left(\Lambda_{2} / \Lambda_{1}\right) \rightarrow H^{k}\left(V / \Lambda_{1}\right) \stackrel{i_{*}}{\rightarrow} H^{k}\left(V / \Lambda_{2}\right) \stackrel{\beta}{\rightarrow} H^{k+1}\left(\Lambda_{2} / \Lambda_{1}\right) \rightarrow \cdots,
\end{aligned}
$$

where $\beta$ is a Bockstein homomorphism.

Proposition 2.11 The inclusion $\Lambda_{1} \stackrel{i}{\hookrightarrow} \Lambda_{2} \rightarrow V$ induces the long exact sequence

$$
\begin{aligned}
& \quad \cdots \stackrel{i_{*}}{\rightarrow} H^{k-2}\left(V / \Lambda_{2}\right) \stackrel{\beta}{\rightarrow} H^{k-1}\left(\Lambda_{2} / \Lambda_{1}\right) \rightarrow \check{H}^{k}\left(\Lambda_{1}\right) \stackrel{\check{i}}{\rightarrow} \check{H}^{k}\left(\Lambda_{2}\right) \rightarrow H^{k}\left(\Lambda_{2} / \Lambda_{1}\right) \\
& \quad \stackrel{\beta}{\rightarrow} H^{k+1}\left(\Lambda_{1}\right) \rightarrow \cdots .
\end{aligned}
$$

Using $i_{*}: H^{k-1}\left(V / \Lambda_{1}\right) \rightarrow H^{k-1}\left(V / \Lambda_{2}\right)$, this can be rewritten as

$$
0 \rightarrow \operatorname{Ker} i_{*} \rightarrow \check{H}^{k}\left(\Lambda_{1}\right) \stackrel{\check{i}}{\rightarrow} \check{H}^{k}\left(\Lambda_{2}\right) \rightarrow \frac{H^{k}\left(\Lambda_{2}\right)}{H^{k}\left(\Lambda_{1}\right)} \rightarrow 0 .
$$

The proof of Proposition 2.11 will follow in a moment. It is based on the homological algebra Lemma 2.13, whose proof is a diagram chase left to the reader. Before giving these details, we wish to highlight the case where $\Lambda_{1}=0$ and $\Lambda_{2}=\Lambda$. From the long exact sequence

$$
\cdots \rightarrow H^{k-1}(\Lambda) \rightarrow H^{k-1}(V) \stackrel{i_{*}}{\rightarrow} H^{k-1}(V / \Lambda) \rightarrow \cdots,
$$


we obtain $\operatorname{Ker} i_{*}=H^{k-1}(V)_{\Lambda}$. Plugging into Proposition 2.11 gives the important exact sequence

$$
0 \rightarrow H^{k-1}(V)_{\Lambda} \rightarrow \check{H}^{k}(0) \rightarrow \check{H}^{k}(\Lambda) \rightarrow H^{k}(\Lambda) \rightarrow 0 .
$$

Remark 2.12 In [3], it is claimed that

$$
0 \rightarrow \operatorname{Ker} i_{*} \rightarrow \check{H}^{k}\left(\Lambda_{1}\right) \rightarrow \check{H}^{k}\left(\Lambda_{2}\right) \rightarrow \Omega_{\Lambda_{2}}^{k} / \Omega_{\Lambda_{1}}^{k} \rightarrow 0
$$

is exact, but Proposition 2.11 shows this is not correct. While there is a surjective homomorphism $H^{k}\left(\Lambda_{2}\right) / H^{k}\left(\Lambda_{1}\right) \rightarrow \Omega_{\Lambda_{2}}^{k} / \Omega_{\Lambda_{1}}^{k}$, it can fail to be injective. When $\Lambda_{1}=0$ and $\Lambda_{2}=\mathbb{Z}$, the relevant map $H^{k}(\mathbb{Z}) \rightarrow \Omega_{\mathbb{Z}}^{k} / \Omega_{0}^{k} \cong H^{k}(\mathbb{R})_{\mathbb{Z}}$ is an isomorphism if and only if $H^{k}(\mathbb{Z})$ is torsion-free.

Lemma 2.13 Assume the following vertical and horizontal sequences are exact.
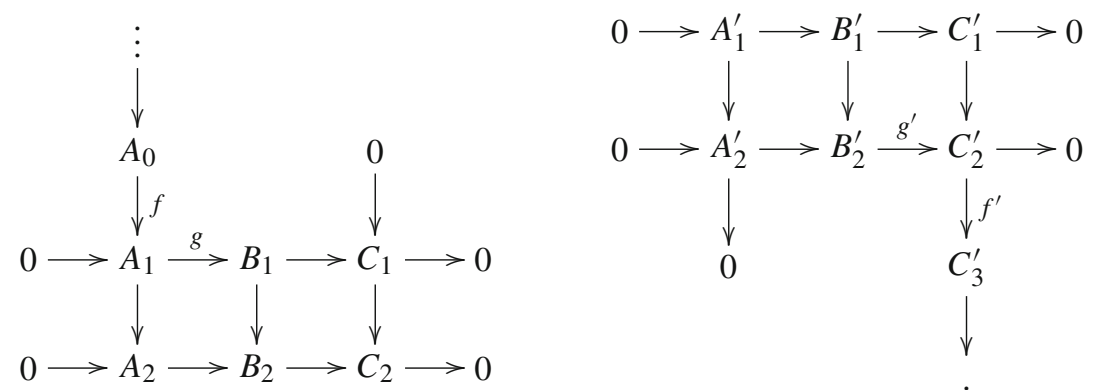

Then, the sequences

$$
\cdots \rightarrow A_{-1} \rightarrow A_{0} \stackrel{g \circ f}{\longrightarrow} B_{1} \rightarrow B_{2} \quad \text { and } \quad B_{1}^{\prime} \rightarrow B_{2}^{\prime} \stackrel{f^{\prime} \circ g^{\prime}}{\longrightarrow} C_{3}^{\prime} \rightarrow C_{4}^{\prime} \rightarrow \cdots
$$

are exact.

Proof The proof is a standard diagram chase and is left to the reader.

Proof (Proof of Proposition 2.11) We will apply Lemma 2.13 to two different complexes, each of which gives half of the desired long exact sequence.

The short exact sequence (2.2) produces the following exact sequences. 


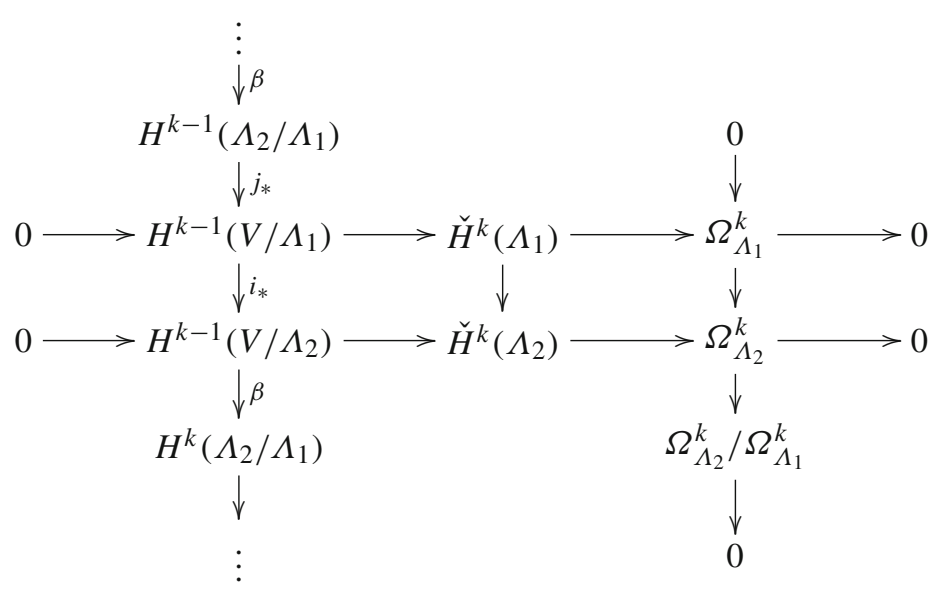

The first case of Lemma 2.13 gives the exact sequence

$\cdots \rightarrow H^{k-2}\left(V / \Lambda_{1}\right) \stackrel{i_{*}}{\rightarrow} H^{k-2}\left(V / \Lambda_{2}\right) \stackrel{\beta}{\rightarrow} H^{k-1}\left(\Lambda_{2} / \Lambda_{1}\right) \rightarrow \check{H}^{k}\left(\Lambda_{1}\right) \rightarrow \check{H}^{k}\left(\Lambda_{2}\right)$

Using the isomorphisms

$$
\frac{H^{k-1}\left(\Lambda_{2} / \Lambda_{1}\right)}{\beta\left(H^{k-2}\left(V / \Lambda_{2}\right)\right)} \cong \frac{H^{k-1}\left(\Lambda_{2} / \Lambda_{1}\right)}{\operatorname{Ker} j_{*}} \cong \text { Image } j_{*} \cong \text { Ker } i_{*},
$$

where $i_{*}: H^{k-1}\left(V / \Lambda_{1}\right) \rightarrow H^{k-1}\left(V / \Lambda_{2}\right)$, we rewrite the exact sequence as

$$
0 \rightarrow \operatorname{Ker} i_{*} \rightarrow \check{H}^{k}\left(\Lambda_{1}\right) \rightarrow \check{H}^{k}\left(\Lambda_{2}\right)
$$

The short exact sequence (2.3) produces the following exact sequences.

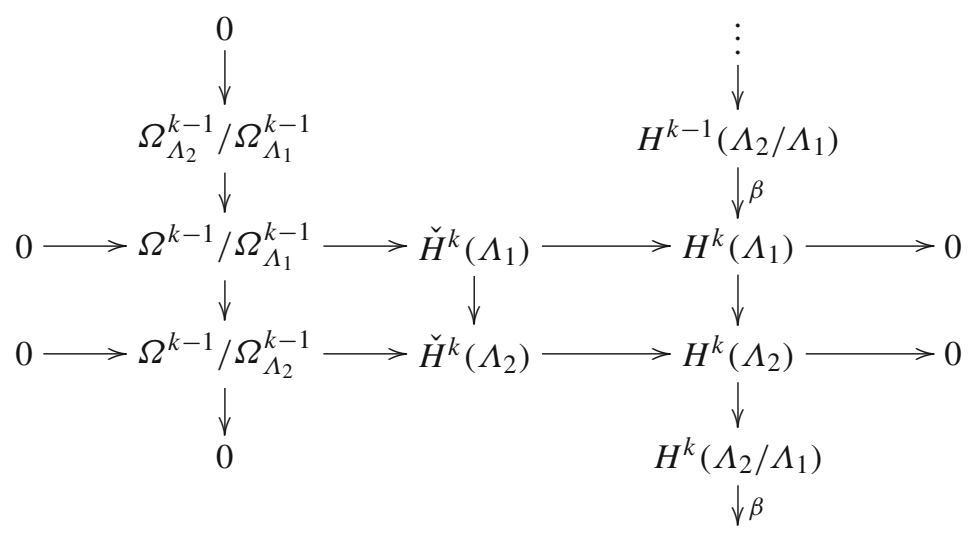


The second case of Lemma 2.13 gives the exact sequence

$$
\check{H}^{k}\left(\Lambda_{1}\right) \rightarrow \check{H}^{k}\left(\Lambda_{2}\right) \rightarrow H^{k}\left(\Lambda_{2} / \Lambda_{1}\right) \stackrel{\beta}{\rightarrow} H^{k+1}\left(\Lambda_{1}\right) \rightarrow \cdots
$$

Using the injective map $\frac{H^{k}\left(\Lambda_{2}\right)}{H^{k}\left(\Lambda_{1}\right)} \rightarrow H^{k}\left(\Lambda_{2} / \Lambda_{1}\right)$, we can rewrite this as

$$
\check{H}^{k}\left(\Lambda_{1}\right) \rightarrow \check{H}^{k}\left(\Lambda_{2}\right) \rightarrow \frac{H^{k}\left(\Lambda_{2}\right)}{H^{k}\left(\Lambda_{1}\right)} \rightarrow 0 .
$$

\section{Trivializations in differential ordinary cohomology}

Trivializations arise whenever there is some notion of objects, morphisms, and a specified trivial object. A trivialization is an isomorphism between an object and the trivial object; an object is trivializable if a trivialization exists. Since all categories in this paper are given by cochain complexes, all trivializations will be of the following form.

Definition 3.1 Let $\left(C^{*}, d\right)$ be a cochain complex with $x \in C^{k}$. The category of trivializations of $x$ in $C^{*}$, denoted $\mathcal{T}(x)$, is the fundamental groupoid

$$
\mathcal{T}(x):=\pi_{\leq 1}\left(C^{k-3} \stackrel{d}{\rightarrow} C^{k-2} \stackrel{d}{\rightarrow} d^{-1}(x)\right)
$$

where $d^{-1}(x) \subset C^{k-1}$ is the preimage of $x$ under $d$.

In other words, an object of $\mathcal{T}(x)$ is a cochain $y \in C^{k-1}$ such that $d y=x$, and the morphisms are given by the additive action of $C^{k-2} / d C^{k-3}$. A trivialization exists if and only if $x \in Z^{k}$ and $[x]=0 \in H^{k}\left(C^{*}\right)$; otherwise, the category is empty. When $x$ is trivializable but non-zero, the set of objects is not naturally a group but instead a torsor over $Z^{k-1}$; i.e. $Z^{k-1}$ acts freely and transitively on $d^{-1}(x)$. Likewise, the set of isomorphism classes

$$
T(x):=\pi_{0}(\mathcal{T}(x))
$$

is a torsor for $Z^{k-1} / d C^{k-2}=H^{k-1}\left(C^{*}\right)$.

Example 3.2 The preimage of a homomorphism occurs as a special case of the above. Let $f: A_{1} \rightarrow A_{2}$ be a homomorphism of abelian groups and $x \in A_{2}$. The category of trivializations $\mathcal{T}(x)$, defined using the complex $0 \rightarrow A_{1} \stackrel{f}{\rightarrow} A_{2}$, has no non-trivial morphisms and is equivalent to the set $T(x)=f^{-1}(x) \subset A_{1}$. 


\subsection{Trivializations of differential cocycles}

We now discuss trivializations of cocycles in differential ordinary cohomology. Again, this idea is not new, and our treatment is heavily influenced by the papers [8] and [4]. Our goal is simply to generalize the fundamental commutative square (2.1) to trivializations. As in Sect. 2, assume $M$ is a smooth manifold, $\Lambda \subset V$ is a totally disconnected subgroup of a vector space, and $\check{C}(*)^{*}(M ; \Lambda)$ is the bi-graded complex of differential cochains.

In the following Definitions 3.3-3.6, let $\check{x}=(c, h, \omega) \in \check{\mathcal{H}}^{k}(M ; \Lambda)$ be a differential cocycle with equivalence class $[\check{x}] \in \check{H}^{k}(M ; \Lambda)$. We simply apply Definition 3.1 to four complexes involving $\check{x}$. While $\check{x}$ has no a priori constraints, the reader should mentally assume its characteristic class vanishes. Proposition 3.9 shows that, in each case, the resulting category of trivializations is non-empty if and only if $[c]=0 \in$ $H^{k}(M ; \Lambda)$.

Definition 3.3 The category of trivializations of the differential cocycle $\check{x} \in$ $\check{\mathcal{H}}^{k}(M ; \Lambda)$ is defined using $\check{C}(k-1)^{*}(M ; \Lambda)$ and is denoted

$$
\mathcal{T}_{\text {geo }}(\check{x}):=\pi_{\leq 1}\left(\check{C}(k-1)^{k-3}(M ; \Lambda) \stackrel{d}{\rightarrow} \check{C}(k-1)^{k-2}(M ; \Lambda) \stackrel{d}{\rightarrow} d^{-1}(\check{x})\right),
$$

where $d^{-1}(\check{x}) \subset \check{C}(k-1)^{k-1}(M ; \Lambda)$. The set of equivalence classes is denoted

$$
T_{\text {geo }}(\check{x}):=\pi_{0}\left(\mathcal{T}_{\text {geo }}(\check{x})\right)
$$

Definition 3.4 The category of trivializations of the characteristic cocycle $c$ is denoted

$$
\mathcal{T}_{\text {top }}(\check{x}):=\mathcal{T}(c)=\pi_{\leq 1}\left(C^{k-3}(M ; \Lambda) \stackrel{\delta}{\rightarrow} C^{k-2}(M ; \Lambda) \stackrel{\delta}{\rightarrow} \delta^{-1}(c)\right)
$$

The set of equivalence classes is denoted

$$
T_{\text {top }}(\check{x}):=\pi_{0}\left(\mathcal{T}_{\text {top }}(\check{x})\right)
$$

Definition 3.5 The set $T_{\text {curv }}(\check{x})=T_{\text {curv }}([\check{x}])$ is the pre-image of $[\check{x}]$ in the homomorphism

$$
\Omega^{k-1}(M ; V) \rightarrow \check{H}^{k}(M ; \Lambda)
$$

defined by (2.3). In other words,

$$
T_{\text {curv }}(\check{x}):=\left\{\eta \in \Omega^{k-1}(M ; V) \mid \int_{\bullet} \eta=\langle[\check{x}], \bullet\rangle \in \operatorname{Hom}\left(Z_{k-1}(M), V / \Lambda\right)\right\},
$$

and elements can be thought of as globally defined connection forms on $M$. 
Definition 3.6 The set $T_{\mathrm{dR}}(\check{x})=T_{\mathrm{dR}}([\check{x}])$ is the pre-image of $[\check{x}]$ in the homomorphism

$$
\check{H}^{k}(M ; 0) \rightarrow \check{H}^{k}(M ; \Lambda)
$$

defined by the inclusion $0 \hookrightarrow \Lambda$; i.e. elements are lifts of the character from $V / \Lambda$ to $V$ :

$$
T_{\mathrm{dR}}(\check{x}):=\left\{\widetilde{\chi} \in \check{H}^{k}(M ; 0) \subset \operatorname{Hom}\left(Z_{k-1}(M), V\right) \mid\langle\widetilde{\chi}, \bullet\rangle=\langle[\check{x}], \bullet\rangle \bmod \Lambda\right\} .
$$

Remark 3.7 Plugging in $\Lambda=0$ to the short exact sequence (2.3) gives a canonical isomorphism

$$
\frac{\Omega^{k-1}(M ; V)}{\Omega^{k-1}(M ; V)_{0}}=\frac{\Omega^{k-1}(M ; V)}{d \Omega^{k-2}(M ; V)} \cong \check{H}^{k}(M ; 0) \text {. }
$$

Let us briefly clarify and justify the definition of $\mathcal{T}_{\text {geo }}(\check{x})$. Because there is a natural equality

$$
\check{Z}(k)^{k}(M ; \Lambda)=\check{Z}(k-1)^{k}(M ; \Lambda),
$$

we can consider trivializations of $\check{x}$ in $\check{C}(k)^{*}(M ; \Lambda)$ or $\check{C}(k-1)^{*}(M ; \Lambda)$. In degree two, this distinction is equivalent to the distinction between flat global sections and arbitrary global sections of a principal $\mathbb{R} / \mathbb{Z}$-bundle with connection. To better visualize the distinction in general, we draw the bi-graded differential cochain complex. The region above the dotted line contains no differential forms.

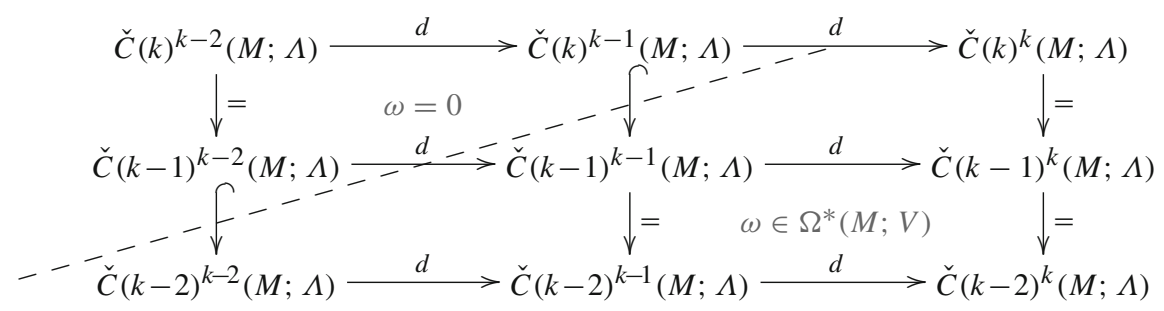

The obstruction to trivializing $\check{x}$ in the middle row is the characteristic class $[\check{x}] \in$ $\breve{H}(k-1)^{k}(M ; \Lambda) \cong H^{k}(M ; \Lambda)$. To trivialize $\check{x}$ in the top row, the differential cohomology class $[\check{x}] \in \check{H}^{k}(M ; \Lambda)$ must vanish. Using $\check{C}(k-1)^{*}(M ; \Lambda)$ is more versatile as it permits trivializations of more general $\check{x}$. And, we can still recover the category of trivializations in $\breve{C}(k)^{*}(M ; \Lambda)$ as the subcategory of flat trivializations in $\mathcal{T}_{\text {geo }}(\check{x})$.

Remark 3.8 Our definition of trivialization is also used in [9], where it is referred to as a "geometric trivialization." Other papers, including [5], use the term "geometric trivialization" for the flat trivializations. We will refrain from using this term so as to avoid confusion. The subscript geo should remind the reader that the equivalence classes contain local geometric data and are non-canonically equivalent to differential 
characters. Similarly, the subscripts top, curv, and dR should convey that these classes generalize the characteristic class, curvature, and de Rham class of a differential character.

Proposition 3.9 For $\check{x}=(c, h, \omega) \in \check{\mathcal{H}}^{k}(M ; \Lambda)$, the sets $T_{\text {geo }}(\check{x}), T_{\text {top }}(\check{x}), T_{\text {curv }}(\check{x})$, and $T_{\mathrm{dR}}(\check{x})$ are all non-empty if and only if the characteristic class $[c]=0 \in$ $H^{k}(M ; \Lambda)$. If $[c]=0$, then these sets are torsors for $\breve{H}^{k-1}(M ; \Lambda), H^{k-1}(M ; \Lambda)$, $\Omega^{k-1}(M ; V)_{\Lambda}$, and $H^{k-1}(M ; V)_{\Lambda}$ respectively.

Proof The categories $\mathcal{T}_{\text {geo }}(\check{x})$ and $\mathcal{T}_{\text {top }}(\check{x})$ are defined using Definition 3.1. The relevant cochain complexes are

$$
\begin{gathered}
\quad . \stackrel{d}{\rightarrow} \check{C}(k-1)^{k-2}(M ; \Lambda) \stackrel{d}{\rightarrow} \check{C}(k-1)^{k-1}(M ; \Lambda) \stackrel{d}{\rightarrow} \check{Z}(k-1)^{k}(M ; \Lambda), \\
\quad \cdots \stackrel{\delta}{\rightarrow} C^{k-2}(M ; \Lambda) \stackrel{\delta}{\rightarrow} C^{k-2}(M ; \Lambda) \stackrel{\delta}{\rightarrow} C^{k-1}(M ; \Lambda) \stackrel{\delta}{\rightarrow} Z^{k}(M ; \Lambda) .
\end{gathered}
$$

Therefore, $T_{\text {geo }}(\check{x})$ is non-empty if and only if

$$
[\check{x}]=0 \in \check{H}(k-1)^{k}(M ; \Lambda) \cong H^{k}(M ; \Lambda),
$$

where we use the isomorphism (2.5). If $[c]=0$, then $T_{\mathrm{geo}}(\check{x})$ is a torsor for

$$
\check{Z}(k-1)^{k-1}(M ; \Lambda) / d \check{C}(k-1)^{k-2}(M ; \Lambda) \cong \check{H}^{k-1}(M ; \Lambda) .
$$

The second cochain complex immediately implies $T_{\text {top }}(\check{x})$ is non-empty if and only if $[c]=0 \in H^{k}(M ; \Lambda)$. If $[c]=0$, then $T_{\text {top }}(\check{x})$ is a torsor for $H^{k-1}(M ; \Lambda)$.

The sets $T_{\text {curv }}(\check{x})$ and $T_{\mathrm{dR}}(\check{x})$ are defined using the construction in Example 3.2, and we rewrite the relevant exact sequences (2.3) and (2.7):

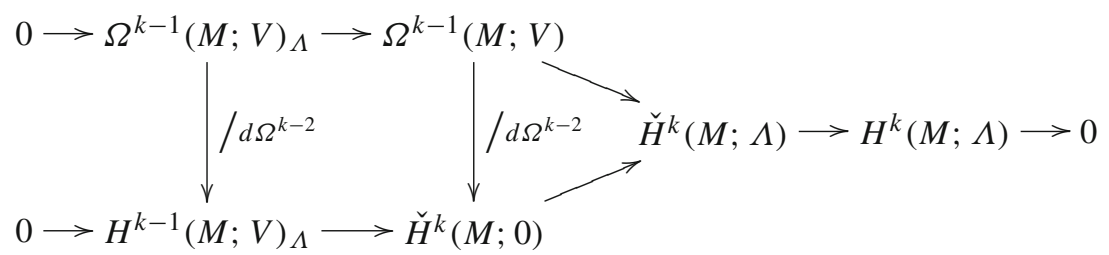

Therefore, $T_{\text {curv }}(\check{x})$ and $T_{\mathrm{dR}}(\check{x})$ are non-empty precisely when the characteristic class $[c]=0 \in H^{k}(M ; \Lambda)$. Assuming $[c]=0$, then $T_{\text {curv }}(\breve{x})$ is a torsor for $\Omega^{k-1}(M ; V)_{\Lambda}$, and $T_{\mathrm{dR}}(\check{x})$ is a torsor for $H^{k-1}(M ; V)_{\Lambda}$.

Proposition 3.10 Let $\check{x}=(c, h, \omega) \in \check{\mathcal{H}}^{k}(M ; \Lambda)$ with $[c]=0 \in H^{k}(M ; \Lambda)$. Then, we have commutative diagrams of categories and sets
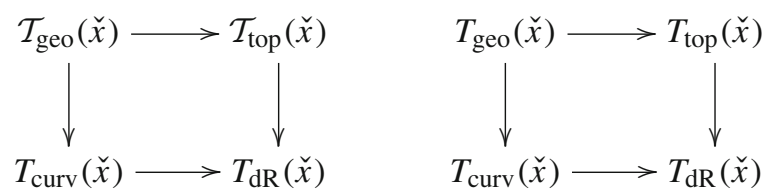
induced by

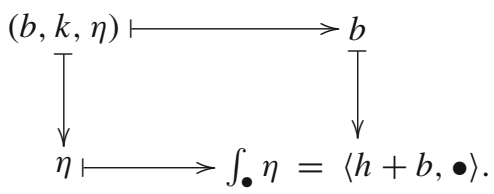

Proof We first establish the maps at the level of objects. Let $\check{s}=(b, k, \eta) \in$ $C^{k-1}(M ; \Lambda) \times C^{k-2}(M ; V) \times \Omega^{k-1}(M)$ be a trivialization of $\check{x}$. Expanding the relation $d \check{s}=\check{x}$, we see

$$
\begin{aligned}
\delta b & =c, \\
\eta-b-\delta k & =h, \\
d \eta & =\omega .
\end{aligned}
$$

Equation (3.2) implies $b \in \mathcal{T}_{\text {top }}(c)$.

Let $z \in Z_{k-1}(M)$. The second equation (3.3), along with the facts $\langle b, z\rangle \in \Lambda$ and $\langle\delta k, z\rangle=\langle k, z\rangle=0$, implies

$$
\begin{aligned}
\int_{z} \eta & =\langle h+b+\delta k, z\rangle \\
& =\langle h, z\rangle \bmod \Lambda .
\end{aligned}
$$

Hence $\eta$ is a global connection form for $\check{x}$; i.e. $\eta \in T_{\text {curv }}(\check{x})$.

Given any $\eta \in T_{\text {curv }}(\check{x})$, we define the class in $T_{\mathrm{dR}}(\check{x}) \subset \check{H}^{k}(M ; 0)$ using the standard map $\Omega^{k-1}(M) \rightarrow \check{H}^{k}(M ; 0)$ given by integration on closed cycles. The maps

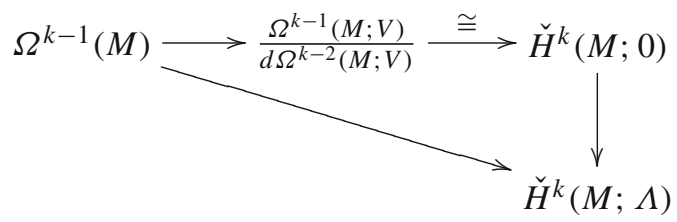

obviously commute, and $\eta \mapsto[\check{x}] \in \check{H}^{k}(M ; \Lambda)$. Therefore, the image of $\eta$ in $\breve{H}^{k}(M ; 0)$ is an element in $T_{\mathrm{dR}}(\check{x})$; integrating $\eta$ lifts the holonomy of $\check{x}$ from $V / \Lambda$ to $V$.

Given $b \in \mathcal{T}_{\text {top }}(\check{x}) \subset C^{k-1}(M ; \Lambda)$, define the character $\widetilde{\chi} \in \check{H}^{k}(M ; 0)$ by

$$
\langle\widetilde{\chi}, \bullet\rangle:=\langle h+b, \bullet\rangle
$$

Since $\langle\chi, \bullet\rangle=\langle h, \bullet\rangle \bmod \Lambda$, then $\widetilde{\chi} \in T_{\mathrm{dR}}(\check{x})$. The commutativity of the square follows from Eq. (3.3). If $z \in Z_{k-1}(M)$, then 


$$
\int_{z} \eta=\langle h+b+\delta k, z\rangle=\langle h+b, z\rangle .
$$

We now define and check the functor on morphisms. Let $\check{s}_{1}=(b, k, \eta) \in$ $\mathcal{T}_{\text {geo }}(\check{x})$, and let $(a, l) \in C^{k-2}(M ; \Lambda) \times C^{k-3}(M ; V)$ determine a morphism in $\operatorname{Hom}_{\mathcal{T}_{\text {geo }}(\check{x})}\left(\check{s}_{1}, \check{s}_{2}\right)$ by $\check{s}_{2}=\check{s}_{1}+d(a, l)$. We then see that

$$
\check{s}_{2}=(b, k, \eta)+d(a, l)=(b+\delta a, k-a-\delta l, \eta) .
$$

The differential form component of $\check{s}_{2}$ still equals $\eta$, so the map from $\mathcal{T}_{\text {geo }}(\check{x}) \rightarrow$ $T_{\text {curv }}(\check{x})$ is well defined.

The functor $\mathcal{T}_{\text {geo }}(\check{x}) \rightarrow \mathcal{T}_{\text {top }}(\check{x})$ is induced by the obvious map of cochain complexes; the morphism $(a, l)$ is sent to $a \in \operatorname{Hom}_{T_{\text {top }}(\check{x})}(b, b+\delta a)$. Finally, the morphism $a$ in $\mathcal{T}_{\text {top }}(\check{x})$ does not change the resulting class in $T_{\mathrm{dR}}(\check{x})$ since

$$
\langle h+b+\delta a, \bullet\rangle=\langle h+b, \bullet\rangle \in \operatorname{Hom}\left(Z_{k-1}(M), V\right) .
$$

The functor $\mathcal{T}_{\text {top }}(\check{x}) \rightarrow T_{\mathrm{dR}}(\check{x})$ is therefore well-defined.

This completes the proof for the commutative diagram of categories. Taking isomorphism classes yields the commutative diagram of sets.

Theorem 3.11 Let $\check{x}=(c, h, \omega) \in \check{\mathcal{H}}^{k}(M ; \Lambda)$ with $[c]=0 \in H^{k}(M ; \Lambda)$. Then, the commutative square on the left is a torsor for the standard commutative square on the right.
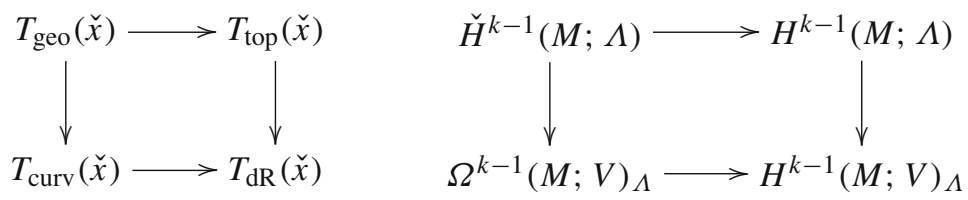

Proof Consider the commutative square of functors from Proposition 3.10 at the level of objects, which we draw below on the left. By Proposition 3.9, each set on the left is a torsor for the corresponding group in the square on the right.
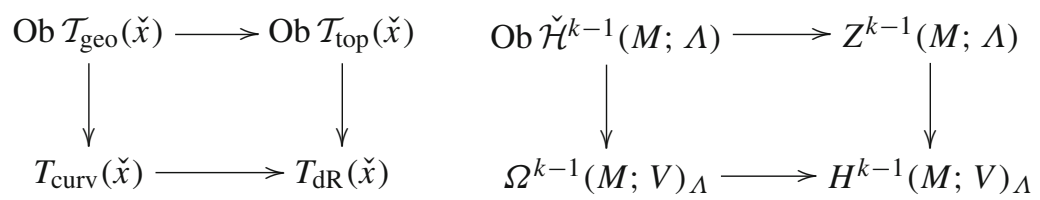
We check that the action is equivariant at the level objects by drawing the action of a cocycle $\left(b^{\prime}, k^{\prime}, \eta^{\prime}\right) \in \check{\mathcal{H}}^{k-1}(M ; \Lambda)$ on $(b, k, \eta) \in \mathcal{T}_{\text {geo }}(\check{x})$ in the following diagram.
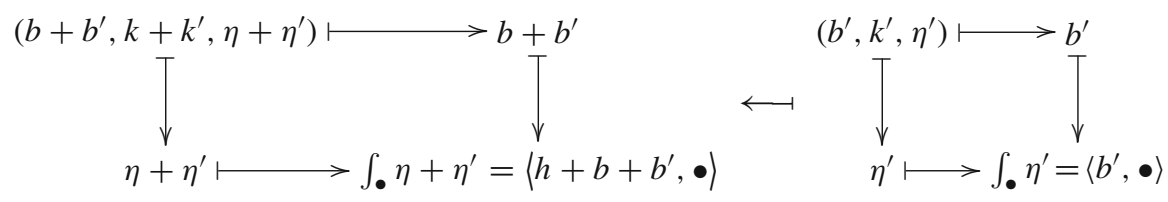

The desired result follows by quotienting $\mathrm{Ob} \mathcal{T}_{\text {geo }}(\check{x})$ and $\mathrm{Ob} \check{\mathcal{H}}^{k-1}(M ; \Lambda)$ by $d \check{C}(k-1)^{k-2}(M ; \Lambda)$, and quotienting $\mathrm{Ob} \mathcal{T}_{\text {top }}(\breve{x})$ and $Z^{k-1}(M ; \Lambda)$ by $d C^{k-2}(M ; \Lambda)$.

Remark 3.12 Note that the categories $\mathcal{T}_{\text {geo }}(\check{x})$ and $\check{\mathcal{H}}^{k-1}(M ; \Lambda)$ are non-canonically isomorphic. The choice of an object in $\mathcal{T}_{\text {geo }}(\check{x})$ determines such an isomorphism. In this sense, we can think of the category $\mathcal{T}_{\text {geo }}(\check{x})$ as being a torsor for the category $\check{\mathcal{H}}^{k-1}(M ; \Lambda)$. More precisely, the objects in $\check{\mathcal{H}}^{k-1}(M ; \Lambda)$ form an abelian group that acts freely and transitively on the objects in $\mathcal{T}_{\text {geo }}(\check{x})$, and both sets of morphisms are naturally bijective. The same statements hold for $\mathcal{T}_{\text {top }}(\check{x})$ and the category of singular cocycles $\mathcal{H}^{k-1}(M ; \Lambda)$.

Theorem 3.11 can be quite useful in practice. First, all maps are surjective. Second, the lack of injectivity is described explicitly by standard short exact sequences. For example, when $H^{k-2}(M ; V / \Lambda)=0$, there is a natural isomorphism $\check{H}^{k-1}(M ; \Lambda) \cong$ $\Omega^{k-1}(M ; V)_{\Lambda}$. In this case, the equivariance in Theorem 3.11 implies that the natural map $T_{\text {geo }}(\check{x}) \rightarrow T_{\text {curv }}(\check{x})$ is a bijection, so a trivialization of $\check{x}$ is completely determined (up to isomorphism) by a differential form. Finally, the sets $T_{\text {curv }}(\check{x})$ and $T_{\mathrm{dR}}(\check{x})$ are defined using only the isomorphism class $[\check{x}] \in \check{H}^{k}(M ; \Lambda)$ as opposed to the cocycle $\check{x} \in \check{\mathcal{H}}^{k}(M ; \Lambda)$.

To explain this last point, we now discuss the dependance of the categories $\mathcal{T}_{\text {geo }}(\check{x})$ and $\mathcal{T}_{\text {top }}(\breve{x})$ on the choice of cocycle $\check{x}$. In examples, one may have a canonical differential cohomology class, but not a canonical cocycle. For example, let $G$ be a compact Lie group with $\lambda \in H^{2 k}(B G ; \mathbb{Z})$. Associated to any principal $G$-bundle with connection $(P, \Theta) \stackrel{\pi}{\rightarrow} M$ is a canonical differential character $\check{\lambda}(P, \Theta) \in \check{H}^{2 k}(M ; \mathbb{Z})$, but there is no canonical differential cocycle representing $\check{\lambda}(P, \Theta)$. In the Hopkins-Singer model, one must choose a classifying map for $P$ and a universal cocycle for $\check{\lambda}$.

Proposition 3.13 Let $\breve{x}$ and $\check{x}+d \check{y}$ be isomorphic objects in $\breve{\mathcal{H}}^{k}(M ; \Lambda)$. Then, the morphism $\check{y}=\left(b^{\prime}, k^{\prime}, 0\right) \in \check{C}(k)^{k-1}(M ; \Lambda)$ determines isomorphisms $\mathcal{T}_{\text {geo }}(\check{x}) \rightarrow$ $\mathcal{T}_{\text {geo }}(\check{x}+d \check{y})$ and $\mathcal{T}_{\text {top }}(\check{x}) \rightarrow \mathcal{T}_{\text {top }}(\check{x}+d \check{y})$ compatible with the maps in Proposition 3.10 and Theorem 3.11 .

Proof The functors are defined in the obvious way

$$
\mathcal{T}_{\text {geo }}(\check{x}) \longrightarrow \mathcal{T}_{\text {geo }}(\check{x}+d \check{y})
$$$$
\mathcal{T}_{\text {top }}(\check{x}) \longrightarrow \mathcal{T}_{\text {top }}(\check{x}+d \check{y})
$$

$\mathrm{Ob}$ :

$$
\check{s} \longmapsto \breve{s}+\check{y}
$$

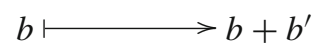

Mor :
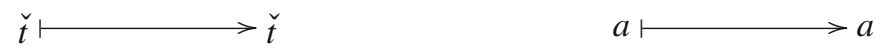
It is straightforward to check compatibility with the characteristic cocycle, curvature, and de Rham maps.

In one sense, Proposition 3.13 says we can discuss trivializations of a differential cohomology class by simply picking a representing cocycle $\check{x} \in \check{\mathcal{H}}^{k}(M ; \Lambda)$. A different choice $\breve{x}^{\prime}$ will give rise to an isomorphic category of trivializations. However, the isomorphism is not canonical! It depends on $\breve{y} \in \check{C}(k)^{k-1}(M ; \Lambda)$, as opposed to only $d \check{y}$. The automorphisms

$$
\operatorname{Aut}(\check{x})=\frac{\check{Z}(k)^{k-1}(M ; \Lambda)}{d \check{C}(k)^{k-2}(M ; \Lambda)}=\check{H}(k)^{k-1}(M ; \Lambda) \cong H^{k-2}(M ; V / \Lambda)
$$

will induce automorphisms of the resulting category $\mathcal{T}_{\text {geo }}(\check{x})$. One must be careful when performing cutting or pasting constructions, or when considering families of differential cohomology classes. On the other hand, these subtleties can be ignored when using the curvature and de Rham classes because the sets $T_{\text {curv }}(\check{x})$ and $T_{\mathrm{dR}}(\check{x})$ only depend on the equivalence class $[\check{x}] \in \check{H}^{k}(M ; \Lambda)$.

\subsection{Hodge decomposition}

We now assume that $M$ is a closed (compact with no boundary) manifold with Riemannian metric $g$. The Hodge Laplacian

$$
\Delta_{g}^{k}=d d^{*}+d^{*} d: \Omega^{k}(M) \rightarrow \Omega^{k}(M)
$$

gives rise to the Hodge orthogonal decomposition of forms

$$
\Omega^{k}(M)=\operatorname{Ker} \Delta_{g}^{k} \oplus d \Omega^{k-1}(M) \oplus d^{*} \Omega^{k+1}(M),
$$

and the canonical inclusion

$$
H^{k}(M ; \mathbb{R}) \cong \operatorname{Ker} \Delta_{g}^{k} \hookrightarrow \Omega^{k}(M)
$$

splits the short exact sequence

$$
0 \longrightarrow d \Omega^{k-1}(M) \longrightarrow \Omega^{k}(M) \stackrel{\text { Hodge }}{\longrightarrow} H^{k}(M ; \mathbb{R}) \longrightarrow 0 .
$$

If $V$ is a vector space, the Hodge decomposition extends to $\Omega^{k}(M ; V)=\Omega^{k}(M) \otimes V$.

Proposition 3.14 Let $(M, g)$ be a closed Riemannian manifold. The Hodge decomposition of forms gives a canonical right inverse to the surjective map $T_{\text {curv }}(\check{x}) \rightarrow$ 
$T_{\mathrm{dR}}(\check{x})$, and it is equivariant with respect to the standard Hodge isomorphism $H^{k-1}(M ; \mathbb{R}) \stackrel{\cong}{\rightrightarrows} \operatorname{Ker} \Delta_{g}^{k-1} \subset \Omega^{k-1}(M)$.

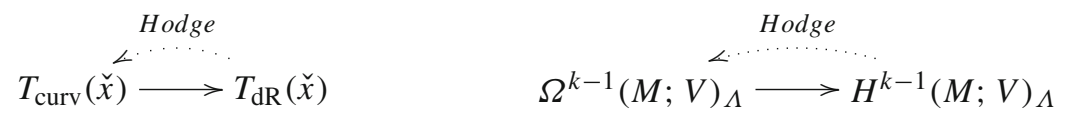

Proof The sets $T_{\text {curv }}(\check{x})$ and $T_{\mathrm{dR}}(\check{x})$ are subsets of $\Omega^{k-1}(M ; V)$ and $\check{H}^{k}(M ; 0)$, respectively, so it suffices to construct a right inverse to $\Omega^{k-1}(M ; V) \rightarrow \check{H}^{k}(M ; 0)$. As mentioned in (3.1), the short exact sequence (2.3) gives an isomorphism

$$
\frac{\Omega^{k-1}(M ; V)}{d \Omega^{k-2}(M ; V)} \cong \check{H}^{k}(M ; 0) .
$$

The Hodge decomposition gives a natural inclusion

$$
\begin{aligned}
\check{H}^{k}(M ; 0) & \cong\left(\Omega^{k-1}(M) \ominus d \Omega^{k-2}(M)\right) \otimes V \\
& =\left(\operatorname{Ker} \Delta_{g}^{k-1} \oplus d^{*} \Omega^{k}(M)\right) \otimes V \subset \Omega^{k-1}(M ; V) .
\end{aligned}
$$

This inclusion maps into the subspace of forms orthogonal to $d \Omega^{k-2}(M ; V)$, so the composition

$$
\check{H}^{k}(M ; 0) \hookrightarrow \Omega^{k-1}(M ; V) \stackrel{/ d \Omega^{k-2}}{\longrightarrow} \check{H}^{k}(M ; 0)
$$

is the identity. Under this inclusion, classes in $\check{H}^{k}(M ; 0)$ represented by closed forms are sent to their harmonic representative.

In other words, to any $\tilde{\chi} \in T_{\mathrm{dR}}(\check{x})$, there is a unique form $\eta \in \Omega^{k-1}(M ; V)$ satisfying both

$$
\int_{\bullet} \eta=\langle\widetilde{\chi}, \bullet\rangle \in \operatorname{Hom}\left(Z_{k-1}(M), V\right) \text { and } d^{*} \eta=0
$$

Combined with Theorem 3.11, this says that when $(M, g)$ is closed Riemannian, there are natural compatible right inverses given by the dotted Hodge arrow in the following commutative squares:
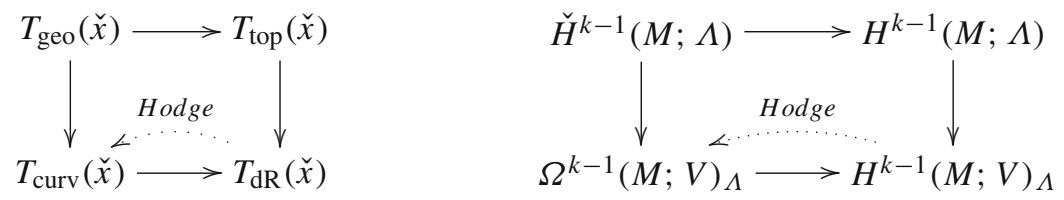
This give an isomorphism of the pullback in the above left square

$$
T_{\text {top }}(\check{x}) \times_{T_{\mathrm{dR}}(\check{x})} T_{\text {curv }}(\check{x}) \cong T_{\text {top }}(\check{x}) \times d \Omega^{k-2}(M ; V) .
$$

Differential characters with harmonic curvature were considered in [6], where they were referred to as harmonic Cheeger-Simons characters. Continuing this analogy, one can define harmonic trivializations as trivializations whose curvature $\eta$ satisfies $d^{*} \eta=0$. For a degree $k$ differential cocycle, the set (of isomorphism classes) of harmonic trivializations is a torsor for the harmonic characters of degree $k-1$.

A natural question is whether the Hodge decomposition can be refined to give canonical right inverses $\check{H}^{k}(M ; \Lambda) \leftarrow H^{k}(M ; \Lambda)$ or $T_{\text {geo }}(\check{x}) \leftarrow T_{\text {top }}(\check{x})$. It is worthwhile to point out that no such construction can exist, as we now explain. We restrict to the category of smooth closed Riemannian manifolds, where the morphisms are isometries (i.e. diffeomorphisms compatible with the Riemannian metric).

Proposition 3.15 For $\Lambda, V \neq 0$, any right splitting of the short exact sequences (2.2), (2.3), or (2.4) will not be functorial in the category of closed Riemannian manifolds.

Proof To prove the non-existence, we construct an explicit counter-example. Since $V$ admits an inclusion $\mathbb{R} \hookrightarrow V$, we only need to check the case $V=\mathbb{R}$. Let $\Lambda \subset \mathbb{R}$ with $\ell \in \Lambda$ and $\ell \neq 0$.

For the short exact sequence (2.2), assume that we have some some construction $\Omega^{k}(-)_{\Lambda} \rightarrow \check{H}^{k}(-; \Lambda)$, denoted by $\omega \mapsto \chi_{\omega}$, that splits $(2.2)$. Let $\mathbb{T}^{k}=\mathbb{R}^{k} / \mathbb{Z}^{k}$ be the $k$-dimensional torus with the flat metric induced from the Euclidean metric on $\mathbb{R}^{k}$, and with the standard coordinates $\left\{x^{i}\right\}$ inherited from $\mathbb{R}^{k}$. Let $\omega=\ell d x^{1} \wedge \ldots \wedge d x^{k}$ be a multiple of the volume form (which is also harmonic), normalized so that $\omega \in$ $\Omega^{k}\left(\mathbb{T}^{k}\right)_{\Lambda}$. Let $f_{a}: \mathbb{T}^{k} \rightarrow \mathbb{T}^{k}$ be an isometry given by translation in the first coordinate; i.e. $f_{a}\left(x^{1}, \ldots, x^{k}\right)=\left(x^{1}+a, x^{2}, \ldots, x^{k}\right)$ where $a \in[0,1]$. Note that $f_{a}^{*} \omega=\omega$. To compare $f_{a}^{*} \chi_{\omega}$ with $\chi_{\omega}$, we evaluate on the $(k-1)$-cycle $0 \times \mathbb{T}^{k-1}$ :

$$
\begin{aligned}
\left\langle f_{a}^{*} \chi_{\omega}-\chi_{\omega},\left[0 \times \mathbb{T}^{k-1}\right]\right\rangle & =\left\langle\chi_{\omega},\left[a \times \mathbb{T}^{k-1}\right]-\left[0 \times \mathbb{T}^{k-1}\right]\right\rangle \\
& =\int_{[0, a] \times \mathbb{T}^{k-1}} \omega=a \ell \bmod \Lambda .
\end{aligned}
$$

For $a \ell \notin \Lambda$, it follows that $f_{a}^{*}\left(\chi_{\omega}\right) \neq \chi_{f_{a}^{*} \omega}$. Hence, $\omega \mapsto \chi_{\omega}$ is not functorial.

A similar argument shows the sequence (2.3) does not naturally split. Let $[c] \in H^{k}\left(\mathbb{T}^{k} ; \Lambda\right)$ be the class mapping to $\ell$ in the homomorphism $H^{k}\left(\mathbb{T}^{k} ; \Lambda\right) \rightarrow$ $H^{k}\left(\mathbb{T}^{k} ; \mathbb{R}\right)$. Since $f_{a}$ is homotopic to the identity map, then $f_{a}^{*}[c]=[c]$. Assume that $\chi_{c} \in \check{H}^{k}\left(\mathbb{T}^{k} ; \Lambda\right)$ is a differential character, with curvature $\omega$, associated to $[c]$. We do not need to assume that $\omega$ is a constant multiple of the volume form. The same argument as above shows that

$$
\left\langle f_{a}^{*} \chi_{c}-\chi_{c},\left[0 \times \mathbb{T}^{k-1}\right]\right\rangle=\int_{[0, a] \times \mathbb{T}^{k-1}} \omega \bmod \Lambda
$$


The above integral must be non-zero for some value of $a$, since

$$
\lim _{a \rightarrow 1} \int_{[0, a] \times \mathbb{T}^{k-1}} \omega=\int_{\mathbb{T}^{k}} \omega=\ell .
$$

Therefore, $f_{a}^{*} \chi_{c} \neq \chi_{c}=\chi_{f_{a}^{*} c}$, and the right splitting of (2.3) cannot be functorial.

Finally, the form $\omega=\ell d x^{1} \wedge \ldots \wedge d x^{k}$ equals $[c]$ in $H^{k}\left(\mathbb{T}^{k} ; \mathbb{R}\right)$. We can combine the above two arguments to show that $f_{a}^{*} \chi_{c, \omega} \neq \chi_{c, \omega}=\chi_{f_{a}^{*} c, f_{a}^{*} \omega}$ for any construction $A^{k}(-; \Lambda) \rightarrow \check{H}^{k}(-; \Lambda)$. Therefore, (2.4) can not split naturally.

Corollary 3.16 There do not exist right inverses $T_{\text {geo }}(-) \leftarrow T_{\text {curv }}(-), T_{\text {geo }}(-) \leftarrow$ $T_{\text {top }}(-)$, or $T_{\text {geo }}(-) \leftarrow T_{\text {top }}(-) \times_{T_{\mathrm{dR}}(-)} T_{\text {curv }}(-)$ that are natural with respect to Riemannian isometries when $\Lambda, V \neq 0$.

Proof By the equivariance from Theorem 3.11, a right inverse

$$
T_{\text {geo }}(-) \longrightarrow T_{\text {curv }}(-)
$$

would induce a functorial splitting of the short exact sequence (2.2). But, this was proved to not exist in Proposition 3.15. For the same reasons, there can not exist functorial right inverses $T_{\text {geo }}(-) \leftarrow T_{\text {top }}(-)$ or $T_{\text {geo }}(-) \leftarrow T_{\text {top }}(-) \times_{T_{\mathrm{dR}}(-)} T_{\text {curv }}(-)$.

\subsection{Compatibility with products}

There is a natural product in $\check{H}^{*}$, and it is defined at the cochain level in [8]. For $\Lambda_{i} \subset V_{i}$, the homomorphism

$$
\check{C}\left(p_{1}\right)^{q_{1}}\left(M ; \Lambda_{1}\right) \otimes \check{C}\left(p_{2}\right)^{q_{2}}\left(M ; \Lambda_{2}\right) \rightarrow \check{C}\left(p_{1}+p_{2}\right)^{q_{1}+q_{2}}\left(M ; \Lambda_{1} \otimes \Lambda_{2}\right)
$$

is defined by the formula

$$
\check{x}_{1} \cdot \check{x}_{2}:=\left(c_{1} \cup c_{2},(-1)^{\left|\omega_{1}\right|} c_{1} \cup h_{2}+h_{1} \cup \omega_{2}+B\left(\omega_{1}, \omega_{2}\right), \omega_{1} \wedge \omega_{2}\right),
$$

where $\Lambda_{1} \otimes \Lambda_{2} \subset V_{1} \otimes V_{2}$. Here $\check{x}_{i}=\left(c_{i}, h_{i}, \omega_{i}\right)$, and $B(\bullet, \bullet)$ is any natural chain homotopy between the cup product $U$ and the wedge product $\wedge$. The product is a graded derivation at the level of cochains,

$$
d\left(\check{x}_{1} \cdot \check{x}_{2}\right)=d \check{x}_{1} \cdot \check{x}_{2}+(-1)^{\left|\check{x}_{1}\right|} \check{x}_{1} \cdot d \check{x}_{2} \in \check{C}(*)^{*}\left(M ; \Lambda_{1} \otimes \Lambda_{2}\right),
$$

so it descends to a homomorphism

$$
\check{H}^{k_{1}}\left(M ; \Lambda_{1}\right) \otimes \check{H}^{k_{2}}\left(M ; \Lambda_{2}\right) \rightarrow \check{H}^{k_{1}+k_{2}}\left(M ; \Lambda_{1} \otimes \Lambda_{2}\right)
$$


In general, this makes $\check{H}^{*}(M ; \Lambda)$ into a (left or right) module over $\check{H}^{*}(M ; \mathbb{Z})$. When $V=\mathbb{R}$ or $\mathbb{C}$ and $\Lambda \subset V$ is a subring, this gives $\breve{H}^{*}(M ; \Lambda)$ a graded commutative ring structure.

For general $\Lambda \subset V$, the product

$$
\check{H}^{k_{1}}(M ; 0) \otimes \check{H}^{k_{2}}(M ; \Lambda) \rightarrow \check{H}^{k_{1}+k_{2}}(M ; 0)
$$

can be described using the isomorphism $\check{H}^{k}(M ; 0) \cong \Omega^{k-1}(M ; V) / d \Omega^{k-2}(M ; V)$ from (3.1). To an equivalence class of $(k-1)$-forms $\left[\eta_{1}\right] \in \check{H}^{k_{1}}(M ; 0)$, and a character $\chi_{2} \in \check{H}^{k_{2}}(M ; \Lambda)$ with curvature $\omega_{2}$, the product is the equivalence class

$$
\left[\eta_{1} \wedge \omega_{2}\right] \in \check{H}^{k_{1}+k_{2}}(M ; 0)
$$

It follows immediately that trivializations and the torsor square from Theorem 3.11 are compatible with the various natural product structures.

Proposition 3.17 Let $\check{x}_{i}=\left(c_{i}, h_{i}, \omega_{i}\right) \in \check{H}^{k_{i}}\left(M ; \Lambda_{i}\right)$ for $i=1$, 2 . If $\check{s}=(b, k, \eta) \in$ $\mathcal{T}_{\text {geo }}\left(\check{x}_{1}\right)$, then

$$
\check{s} \cdot \check{x}_{2} \in \mathcal{T}_{\text {geo }}\left(\check{x}_{1} \cdot \check{x}_{2}\right)
$$

The characteristic cocycle of $\breve{s} \cdot \check{x}_{2}$ is $b \cup c_{2}$, the curvature is $\eta \wedge \omega_{2}$, and the de Rham class is given by the product (3.5).

Proof Using Eq. (3.4) and the assumption that $d \check{s}=\check{x}_{1}$, we have

$$
d\left(\check{s}^{\prime} \cdot \check{x}_{2}\right)=d \check{s} \cdot \check{x}_{2}+(-1)^{k} \check{s}^{2} \cdot d \check{x}_{2}=\check{x}_{1} \cdot \check{x}_{2}
$$

The characteristic cocycle and curvature follow from the definition of $\check{s}^{\prime} \check{x}_{2}$. The de Rham class is the equivalence class of the curvature $\eta \wedge \omega_{2}$ modulo $d \Omega^{k_{1}+k_{2}-2}(M ; V)$, which is precisely the product (3.5).

\subsection{Compatibility with integration}

Hopkins-Singer also define the notion of an $\check{H}$-orientation for a smooth map $f: X \rightarrow$ $B$ of relative dimension $n$. The details can be found in Sections 2.4 and 3.4 of [8], but it amounts to factoring $f$ through an embedding $X \hookrightarrow B \times \mathbb{R}^{N}$ and choosing a differential Thom cocycle for the normal bundle. Such a cocycle (with fiberwise compact support) $\breve{U} \in \check{\mathcal{H}}_{c}^{N-n}(v ; \mathbb{Z})$ is a differential refinement of a Thom class for the normal bundle $v \rightarrow X$. When $f: X \rightarrow B$ is a smooth fiber bundle whose fibers are diffeomorphic to a closed manifold $M$, an orientation and Riemannian metric on $M$ define an $\check{H}$-orientation up to isomorphism. 
Given an $\check{H}$-oriented map $f: X \rightarrow B$, of relative dimension $n$, the usual pushforward construction in cohomology extends to the differential cochain complex and is given by the composition

$$
\begin{aligned}
& \check{C}(p)^{q}(X ; \Lambda) \stackrel{\pi^{*} \cup \check{U}}{\longrightarrow} \check{C}(p+N-n)_{c}^{q+N-n}(\nu ; \Lambda) \rightarrow \check{C}(p+N-n)_{c}^{q+N-n}\left(B \times \mathbb{R}^{N} ; \Lambda\right) \\
& \quad \stackrel{\int_{\mathbb{R}^{N}}^{\longrightarrow}}{\longrightarrow} \check{C}(p-n)^{q-n}(B ; \Lambda) .
\end{aligned}
$$

Here, $\int_{\mathbb{R}^{N}}$ is defined using the slant product on singular cochains and integration on forms. The composition, in both the differential cochain and singular cochain complex, is referred to as integration and is denoted

$$
\begin{gathered}
\int_{X / B}: \check{C}(p)^{q}(X ; \Lambda) \longrightarrow \check{C}(p-n)^{q-n}(B ; \Lambda), \\
\int_{X / B}: C^{q}(X ; \Lambda) \longrightarrow C^{q-n}(B ; \Lambda) .
\end{gathered}
$$

This is compatible with the usual integration of differential forms; i.e. if $\check{x} \in$ $\check{\mathcal{H}}^{k}(X ; \Lambda)$, then

$$
\operatorname{curv}\left(\int_{X / B} \check{x}\right)=\int_{X / B} \operatorname{curv}(\check{x}) \in \Omega^{k-n}(B ; V)
$$

where the right-hand integral takes place in the de Rham complex.

The integration functor satisfies a generalized Stokes formula

$$
d \int_{X / B} \check{x}=\int_{X / B} d \check{x}+(-1)^{|\check{x}|-n} \int_{X / B} \check{x}
$$

Assuming the fibers of $f$ have no boundary, the Stokes formula implies $\int_{X / B}$ is a cochain map and induces homomorphisms

$$
\int_{X / B}: \check{H}^{k}(X ; \Lambda) \longrightarrow \check{H}^{k-n}(B ; \Lambda)
$$

compatible with the usual pushforward in cohomology. When $\Lambda=0$, this homomorphism is given by integrating equivalence classes of forms:

$$
\int_{X / B}: \check{H}^{k}(X ; 0) \cong \frac{\Omega^{k-1}(X ; V)}{d \Omega^{k-2}(X ; V)} \stackrel{\int_{X / B}}{\longrightarrow} \frac{\Omega^{k-n-1}(B ; V)}{d \Omega^{k-n-2}(B ; V)} \cong \check{H}^{k-n}(B ; 0) .
$$

It is straightforward to check that trivializations behave well with respect to integration. 
Proposition 3.18 Suppose that $f: X \rightarrow B$ is an $\check{H}$-oriented map of relative dimension $n$ and whose fibers have no boundary. If $\check{x} \in \check{\mathcal{H}}^{k}(X ; \Lambda)$ admits a trivialization, then integration induces a functor

$$
\int_{X / B}: \mathcal{T}_{\mathrm{geo}}(\check{x}) \longrightarrow\left(\int_{X / B} \check{x}\right)
$$

that is compatible with the various forms of integration. More precisely, the following diagram containing the two torsor squares commutes; the diagonal arrows are induced by the integrations (3.6), (3.7), (3.10), and the ordinary integration of differential forms.

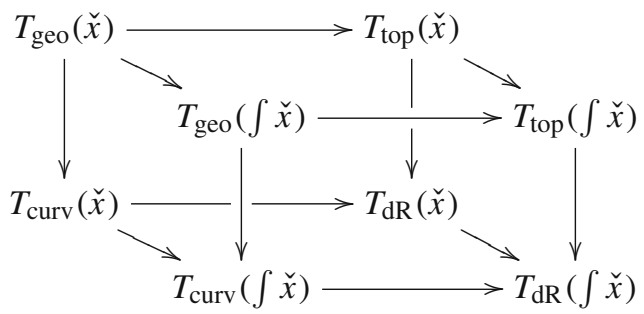

Proof The Stokes formula implies that if $d \check{s}=\check{x}$, then $d \int_{X / B} \check{s}=\int_{X / B} \check{x}$. If $d \check{y}=\check{s}_{2}-\check{s}_{1}$, then $d \int_{X / B} \check{y}=\int_{X / B} \check{s}_{2}-\int_{X / B} \check{s}_{1}$. Therefore, the integration in (3.6) induces a functor $\mathcal{T}_{\text {geo }}(\check{x}) \rightarrow \mathcal{T}_{\text {geo }}\left(\int_{X / B} \check{x}\right)$. The commutativity of the diagram is an immediate consequence of the the fact that integration in the differential cochain complex commutes with integration in the singular and de Rham complexes.

\section{Trivializing circle bundles}

The category $\check{\mathcal{H}}^{2}(M ; \mathbb{Z})$ is equivalent to the category of principal $\mathbb{R} / \mathbb{Z}$-bundles with connection. ${ }^{3}$ We now explain the relationship between trivializations of principal $\mathbb{R} / \mathbb{Z}$ bundles and the torsor square for trivializations of degree two classes.

Begin by fixing a principal $\mathbb{R} / \mathbb{Z}$-bundle $P \stackrel{\pi}{\rightarrow} M$ with connection $\Theta$. A trivialization of $P$, not necessarily preserving the connection, is defined as a bundle isomorphism $P \cong M \times \mathbb{R} / \mathbb{Z}$; this is naturally equivalent to a global section $p \in C^{\infty}(M, P)$. The bundle $P$ is trivializable if and only if its characteristic class $c(P)=0 \in H^{2}(M ; \mathbb{Z})$. Assuming $c(P)=0$, the set of global sections naturally fits into the following commutative square.

\footnotetext{
3 We use $\mathbb{R} / \mathbb{Z}$ instead of $U(1)$ so that all group structures are written additively and to eliminate factors of $i$ and $2 \pi$.
} 


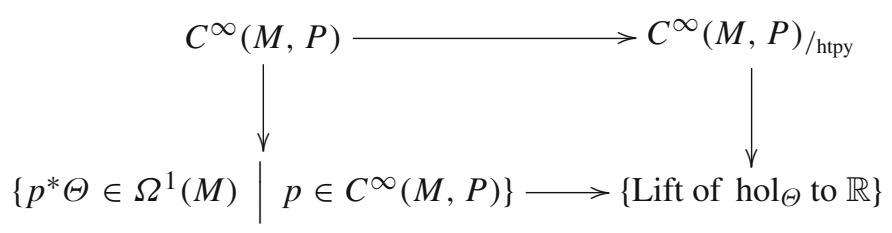

The top right corner consists of global sections modulo homotopy through the space of sections. The bottom left corner consists of all globally defined connection 1-forms on $M$ for the connection $\Theta$. The bottom right corner consists of all lifts of the holonomy from $\mathbb{R} / \mathbb{Z}$ to $\mathbb{R}$ that are compatible with the curvature $d \Theta$.

The abelian group $C^{\infty}(M, \mathbb{R} / \mathbb{Z})$, with product given by pointwise addition in $\mathbb{R} / \mathbb{Z}$, acts freely and transitively on the space of global sections. For $p \in C^{\infty}(M, P)$ and $f \in C^{\infty}(M, \mathbb{R} / \mathbb{Z})$, the resulting section is defined by

$$
(p+f)(x):=p(x)+f(x) .
$$

Pulling back the connection along the section $p+f$ results in the change

$$
(p+f)^{*} \Theta=p^{*} \Theta+d f
$$

We do not need to include the adjoint action because $\mathbb{R} / \mathbb{Z}$ is abelian.

Any two lifts of $\operatorname{hol}_{\Theta}$ to $\mathbb{R}$ will differ by an element in $\operatorname{Hom}\left(H_{1}(M), \mathbb{Z}\right)$. A priori, one may think they only need to differ by an element in $\operatorname{Hom}\left(Z_{1}(M), \mathbb{Z}\right)$. We assume, though, that our lifts are "smooth" in the sense that integrating the curvature form calculates the difference between the $\mathbb{R}$-valued holonomies of homotopic paths. More concisely, the lift is an element in $\check{H}^{2}(M ; 0)$.

It follows that the commutative square (4.1) is a torsor over the following commutative square, which was explained in Example 2.6 to be canonically isomorphic to the standard commutative square for $\check{H}^{1}(M ; \mathbb{Z})$.

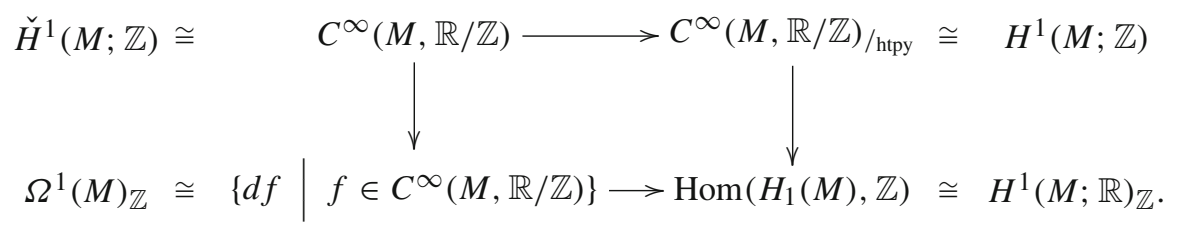

The bottom right isomorphism was proven in Lemma 2.10.

We wish to show that the square (4.1) is equivalent to the square from Theorem 3.11. We should first explain the equivalence, discussed in Example 2.7 of [8], between the category of principal $\mathbb{R} / \mathbb{Z}$-bundles with connection on $M$ and the category $\check{\mathcal{H}}^{2}(M ; \mathbb{Z})$. The key idea is that a bundle is determined completely by its local sections, and the connection $\Theta$ is determined by local connection forms $\nabla$.

To any $\mathbb{R} / \mathbb{Z}$-bundle with connection $(P, \Theta) \stackrel{\pi}{\rightarrow} M$, one can associate its sheaf of sections: to the open set $U \subset M$ is assigned $C^{\infty}(U, P)$, which is a torsor for 
$C^{\infty}(U, \mathbb{R} / \mathbb{Z})$ when it is non-empty. The connection $\Theta$ determines local connection 1-forms, or maps

$$
\nabla: C^{\infty}(U, P) \rightarrow \Omega^{1}(U)
$$

that transform according to the previously mentioned rule $\nabla^{p+f}=\nabla^{p}+d f$. A morphism of $(P, \Theta)$ induces a morphism of the resulting sheaf $C^{\infty}(-, P)$ that preserves the connection $\nabla$.

Similarly, to a differential cocycle $\check{x} \in \check{\mathcal{H}}^{2}(M ; \mathbb{Z})$ one can consider the sheaf of trivializations

$$
U \mapsto T_{\text {geo }}\left(\left.\check{x}\right|_{U}\right)
$$

When $T_{\text {geo }}\left(\left.\check{x}\right|_{U}\right)$ is non-empty, it is a torsor for $\check{H}^{1}(U ; \mathbb{Z}) \cong C^{\infty}(U, \mathbb{R} / \mathbb{Z})$, and $T_{\text {geo }}\left(\left.\check{x}\right|_{U}\right) \rightarrow T_{\text {curv }}\left(\left.\check{x}\right|_{U}\right)$ gives a local connection form $\nabla$ transforming by $\nabla^{\check{s}+f}=$ $\nabla^{\check{s}}+d f$.

Now, we assume that $(P, \Theta) \stackrel{\pi}{\rightarrow} M$ is represented by $\check{x} \in \check{\mathcal{H}}^{2}(M ; \mathbb{Z})$ in the sense that the resulting sheaf of sections with connection are equal, or that we have chosen an isomorphism between them.

Proposition 4.1 If $(P, \Theta) \stackrel{\pi}{\rightarrow} M$ is represented by $\check{x} \in \check{\mathcal{H}}^{2}(M ; \mathbb{Z})$, then the commutative square (4.1) is in natural bijection with the commutative square

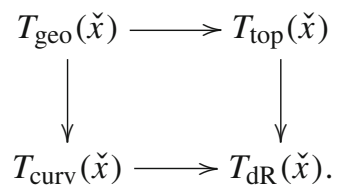

Proof We assume $c(P)=0$, since all of the relevant spaces are empty otherwise. An element in $T_{\text {geo }}(\check{x})$ is precisely the choice of a global section of the sheaf

$$
\left(U \mapsto T_{\mathrm{geo}}\left(\left.\check{x}\right|_{U}\right)\right) \cong\left(U \mapsto C^{\infty}(U, P)\right)
$$

which is equivalent to a global section $p \in C^{\infty}(M, P)$. The sheaves of local sections/trivializations are equivariant with respect to the action of $C^{\infty}(-, \mathbb{R} / \mathbb{Z}) \cong$ $\breve{H}^{1}(-; \mathbb{Z})$, so the bijection

$$
T_{\text {geo }}(\check{x}) \longleftrightarrow C^{\infty}(M, P)
$$

is also equivariant. The squares (4.1) and (4.2) are both torsors over the square for $\breve{H}^{1}(M ; \mathbb{Z})$, and we have an equivariant map between the top-left corner of both squares.

While bijections between the remaining corners can be constructed explicitly, Lemma 4.2 immediately implies their existence and uniqueness. 
Lemma 4.2 Let $G_{1} \rightarrow G_{2}$ be a surjective group homomorphism. Suppose $A_{i}$ and $B_{i}$ are (left) torsors over the groups $G_{i}$, and there are $G_{1} \rightarrow G_{2}$ equivariant maps $A_{1} \stackrel{\alpha}{\rightarrow} A_{2}$ and $B_{1} \stackrel{\beta}{\rightarrow} B_{2}$. Then, a $G_{1}$-equivariant map $A_{1} \stackrel{f_{1}}{\rightarrow} B_{1}$ induces a unique $G_{2}$ equivariant map $A_{2} \stackrel{f_{2}}{\rightarrow} B_{2}$.

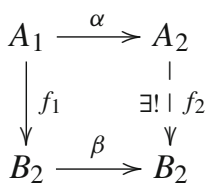

Proof For $a_{2} \in A_{2}$, choose some element $a_{1} \in \alpha^{-1}\left(a_{2}\right) \subset A_{1}$. Define $f_{2}\left(a_{2}\right):=$ $\beta\left(f_{1}\left(a_{1}\right)\right)$. This is well-defined since any other choice $a_{1}^{\prime}=g_{0} a_{1}$ for a unique $g_{0} \in$ $\operatorname{Ker}\left(G_{1} \rightarrow G_{2}\right)$, and

$$
\beta\left(f_{1}\left(g_{0} a_{1}\right)\right)=\beta\left(g_{0} f_{1}\left(a_{1}\right)\right)=\beta\left(f_{1}\left(a_{1}\right)\right) .
$$

The $G_{2}$-equivariance of $f_{2}$ follows by a similar argument. If $g_{1} \mapsto g_{2}$, then

$$
f_{2}\left(g_{2} a_{2}\right)=\beta\left(f_{1}\left(g_{1} a_{1}\right)\right)=\beta\left(g_{1} f_{1}\left(a_{1}\right)\right)=g_{2} f_{2}\left(a_{2}\right) .
$$

Finally, we remark how Propositions 3.18 and 4.1 can combine in a useful way. Suppose that $X, B$ are smooth manifolds without boundary, that

$$
X \stackrel{f}{\longrightarrow} B
$$

is an $\check{H}$-oriented map of relative dimension $n$, and that we have a principal $\mathbb{R} / \mathbb{Z}$-bundle with connection $(P, \Theta) \rightarrow B$. If we can represent this bundle by $\int_{X / B} \check{x} \in \check{\mathcal{H}}^{2}(B ; \mathbb{Z})$ for some $\check{x} \in \check{\mathcal{H}}^{n+2}(X ; \mathbb{Z})$, it may be more convenient to trivialize the bundle on $B$ by trivializing $\check{x}$ in $X$.

We first make a couple of remarks about trivializing $\mathbb{R} / \mathbb{Z}$-bundles with connection. There is no torsion in the cohomology group $H^{1}(B ; \mathbb{Z})$, so the homomorphism $H^{1}(B ; \mathbb{Z}) \rightarrow H^{1}(B ; \mathbb{R})_{\mathbb{Z}}$ is an isomorphism. This implies that lifting the holonomy to $\mathbb{R}$ is equivalent to specifying the homotopy class of a global section. Also, the choice of a global connection form on $B$ determines a trivial connection on $P$ by $\Theta-\pi^{*} p^{*} \Theta \in \Omega^{1}(P)$. On each connected component of $B$, the choice of a trivial connection only determines a global section up to a constant $\mathbb{R} / \mathbb{Z}$-phase. Algebraically, this is explained by the fact that $\check{H}^{1}(B ; \mathbb{Z}) \rightarrow \Omega^{1}(B)_{\mathbb{Z}}$ has non-trivial kernel $H^{0}(B ; \mathbb{R} / \mathbb{Z})$.

Assume that $\int_{X / B} \check{x}$ represents $(P, \Theta) \stackrel{\pi}{\rightarrow} B$. By Propositions 3.18 and 4.1, a trivialization $\check{s} \in T_{\text {geo }}(\check{x})$ induces a global section of $P$ by $\int_{X / B} \check{s} \in T_{\text {geo }}\left(\int_{X / B} \check{x}\right)$. The characteristic class of $\breve{s}$ determines the homotopy class of the induced global section, the curvature of $\check{s}$ induces a trivial connection on $P$, and the de Rham class of 
$\check{s}$ lifts the holonomies of $(P, \Theta)$ to $\mathbb{R}$. While it may be unreasonable to construct $\check{x}$ and $\check{s}$ explicitly in $\check{C}(*)^{*}(X ; \mathbb{Z})$, it is likely that one can define the differential character $[\check{x}]$ and the curvature of a trivialization $\breve{s}$. In practice, this may be all that one needs. If $H^{n}(X ; \mathbb{R} / \mathbb{Z})=0$, then $T_{\text {geo }}(\check{x}) \rightarrow T_{\text {curv }}(\check{x})$ is a bijection. More generally, if the integration homomorphism $H^{n}(X ; \mathbb{R} / \mathbb{Z}) \rightarrow H^{0}(B ; \mathbb{R} / Z)$ is zero, then $T_{\text {curv }}(x) \rightarrow$ $T_{\text {curv }}\left(\int_{X / B} \check{x}\right)$ lifts to $T_{\text {curv }}(x) \rightarrow T_{\text {geo }}\left(\int_{X / B} \check{x}\right)$. In this case, a global section of $P$ can be completely determined by a differential form on $X$, whereas it could not be determined solely by a form on $B$.

Acknowledgments The author wishes to thank both Michigan State University, where most of this research was originally conducted, and the Hausdorff Research Institute for Mathematics, where most of this paper was written. Thanks also to Alex Kahle, Arturo Prat-Waldron, Konrad Waldorf, Fei Han, Peter Teichner, and an anonymous referee for helpful comments.

\section{References}

1. Brylinski, J.L.: Loop Spaces, Characteristic Classes and Geometric Quantization, Progress in Mathematics, vol. 107. Birkhäuser Boston Inc., Boston (1993)

2. Bunke, U., Schick, T.: Smooth $K$-theory. Astérisque (328), 45-135 (2010) (2009)

3. Cheeger, J., Simons, J.: Differential characters and geometric invariants. In: Geometry and Topology (College Park, Md., 1983/1984), Lecture Notes in Math., vol. 1167, pp. 50-80. Springer, Berlin (1985)

4. Freed, D.S.: Dirac charge quantization and generalized differential cohomology. In: Surveys in Differential Geometry, Surv. Differ. Geom., vol. VII, pp. 129-194. Int. Press, Somerville (2000)

5. Freed, D.S., Moore, G.W.: Setting the quantum integrand of M-theory. Commun. Math. Phys. 263(1), 89-132 (2006)

6. Green, R., Mathai, V.: Harmonic Cheeger-Simons characters with applications. J. Geom. Phys. 59(5), 663-672 (2009)

7. Harvey, R., Lawson, B.: From sparks to grundles-differential characters. Comm. Anal. Geom. 14(1), 25-58 (2006)

8. Hopkins, M.J., Singer, I.M.: Quadratic functions in geometry, topology, and M-theory. J. Differ. Geom. 70(3), 329-452 (2005)

9. Kahle, A., Valentino, A.: T-duality and differential K-theory (2009) [arXiv:0912.2516]

10. Simons, J., Sullivan, D.: Axiomatic characterization of ordinary differential cohomology. J. Topol. 1(1), 45-56 (2008)

11. Waldorf, K.: A loop space formulation for geometric lifting problems. J. Aust. Math. Soc. 90(1), 129-144 (2011)

12. Waldorf, K.: String connections and Chern-Simons theory. Trans. Am. Math. Soc. 365(8), 4393-4432 (2013). 